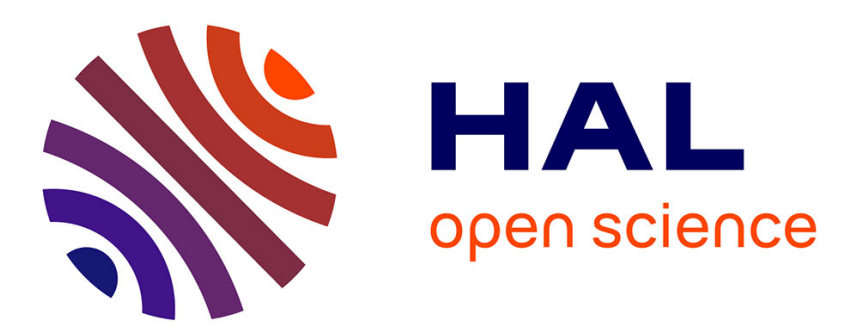

\title{
A new biophysical decompression model for estimating the risk of articular bends during and after decompression
}

\author{
J. Hugon, J-C. Rostain, B. Gardette
}

\section{- To cite this version:}

J. Hugon, J-C. Rostain, B. Gardette. A new biophysical decompression model for estimating the risk of articular bends during and after decompression. Journal of Theoretical Biology, 2011, 283 (1), pp.168. 10.1016/j.jtbi.2011.05.002 . hal-00719497

\section{HAL Id: hal-00719497 \\ https://hal.science/hal-00719497}

Submitted on 20 Jul 2012

HAL is a multi-disciplinary open access archive for the deposit and dissemination of scientific research documents, whether they are published or not. The documents may come from teaching and research institutions in France or abroad, or from public or private research centers.
L'archive ouverte pluridisciplinaire HAL, est destinée au dépôt et à la diffusion de documents scientifiques de niveau recherche, publiés ou non, émanant des établissements d'enseignement et de recherche français ou étrangers, des laboratoires publics ou privés. 


\section{Author's Accepted Manuscript}

A new biophysical decompression model for estimating the risk of articular bends during and after decompression

J. Hugon, J-C. Rostain, B. Gardette

PII:

S0022-5193(11)00236-0

DOI: doi:10.1016/j.jtbi.2011.05.002

Reference: YJTBI 6466

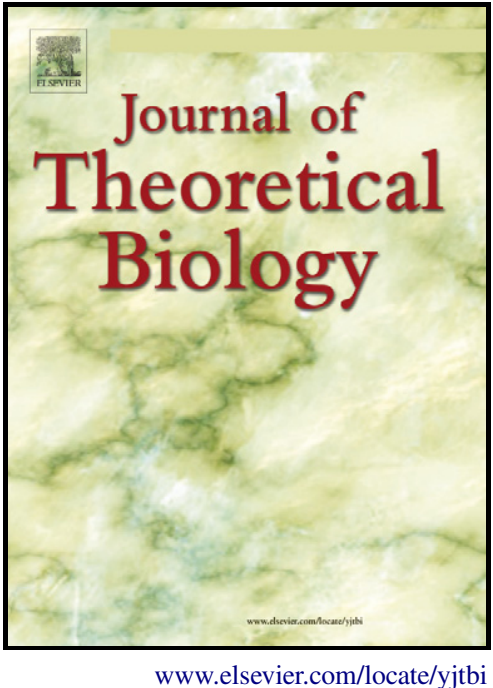

To appear in: $\quad$ Journal of Theoretical Biology

Received date: $\quad 25$ July 2010

Revised date: 29 April 2011

Accepted date: $\quad 3$ May 2011

Cite this article as: J. Hugon, J-C. Rostain and B. Gardette, A new biophysical decompression model for estimating the risk of articular bends during and after decompression, Journal of Theoretical Biology, doi:10.1016/j.jtbi.2011.05.002

This is a PDF file of an unedited manuscript that has been accepted for publication. As a service to our customers we are providing this early version of the manuscript. The manuscript will undergo copyediting, typesetting, and review of the resulting galley proof before it is published in its final citable form. Please note that during the production process errors may be discovered which could affect the content, and all legal disclaimers that apply to the journal pertain. 
A new biophysical decompression model for estimating the risk of articular bends during and after decompression

\author{
J. HUGON
}

Eng, MSc, Université de la Méditerranée, UMR - MD2, P2COE, Institut de Neuroscience J. Roche, Faculté de Médecine Nord, 13916 Marseille Cedex 20 France

Corresponding author ; Tel : +33(0)492926951; Fax : +33(0)492926970 ; e-mail hugon.julien@gmail.com

\author{
J-C. ROSTAIN
}

PhD, DSc, Université de la Méditerranée, UMR - MD2, P2COE, Institut de Neuroscience J. Roche, Faculté de Médecine Nord, 13916 Marseille Cedex 20 France Tel : +33(0)491698906; Fax : +33(0)491653851;e-mail jcrostain@univmed.fr

\title{
B. GARDETTE
}

PhD, DSc, COMEX SA 36 bd des Océans 13275 MARSEILLE Cedex 9 - France Tel : +33 (0)491297500; Fax : +33(0)491297507; e-mail comexsa@,comex.fr

Included

7 Figures

5 Tables

7479 words

Abstract 314 words

40 references 


\begin{abstract}
The biophysical models that intend to predict the risk of decompression sickness after a change of pressure are not numerous. Few approaches focus in particular on joints as target tissues, with the aim to describe properly the mechanisms inducing pain. Nevertheless, for this type of decompression incidents, called articular bends, no model proved to fit the empirical results for a broad range of exposures and decompression procedures. We present here an original biophysical decompression model for describing the occurrence of articular bends. A target joint is broken down into two parts that exchange inert gases with the blood by perfusion and with each other by diffusion over distances of a few millimeters. This diffusion pathway allows the slow amplification of microbubbles growing during and after decompression, consistent with the possible delayed occurrence of bends. The diffusion coefficients introduced into this model are larger than those introduced into most modern decompression models. Their value remains physical $\left(\# 10^{-9} \mathrm{~m}^{2} / \mathrm{s}\right)$. Inert gas exchanges and the formation, amplification and resorption of microbubbles during and after decompression were simulated. We used a critical gas volume criterion for predicting the occurrence of bends. A risk database extracted from COMEX experience and other published studies was used for the correlation of model parameters not known a priori. We considered a large range of exposure, and the commonly used inert gases nitrogen and helium. This correlation phase identified the worst biophysical conformations most likely to lead to the formation, in tissues such as tendons, of a large number of microbubbles recruited from pre-existing gas nuclei during decompression. The risk of bends occurrence was found to be linked to the total separated gas volume generated during and after decompression. A clamping phenomenon occurs soon after the start of decompression, greatly slowing the gas exchanges controlled especially by the oxygen window. This model, which reproduces many empirical findings, may be considered both descriptive and predictive.
\end{abstract}

Key words: decompression sickness, prevention, bubbles, articulation, modeling 


\section{Introduction}

Little is known about the biophysical mechanisms underlying the effects on the body of decompression stress, despite a century of research. The scientific community agrees on only one point: the bubbles formed in the body during decompression may be pathogenic. Pol and Watelle (26) were the first to propose this hypothesis, which was later also put forward by Bert (2). The only known way to prevent decompression sickness is to slow decompression, thereby limiting the production of bubbles.

At the request of the Royal Navy, Haldane (5) subsequently described a physiological basis for decompression problems. He produced procedures and tables based on a simple formula assuming that the body consists of $\mathrm{N}$ independent compartments exchanging inert gases with the blood by perfusion. The criterion for preventing the occurrence of symptoms was selected on the basis of the experimental results obtained: a pressure ratio acceptable for the body. The mechanisms of microbubbles formation, about much very little was known at the time, were not taken into account. However, when modelling or representing decompression mechanisms, it is also important to consider the conditions favouring the formation of microbubbles within the body. The theory of nucleation has progressed since the work of Harvey $(12,13)$ and has provided new insight driving changes in procedures from the days of Haldane. For example, the introduction of deeper decompression stages to confine the microbubbles formed from recruited micronuclei, by limiting their size and growth rate, may be considered a step forward, although this procedure remains controversial $(23,4)$.

For more detailed decompression modelling, mechanisms must be found to account for the occurrence of a given decompression incident. The sites and conditions of the nucleation that characterize that incident must be investigated.

Minor incidents, affecting the joints or skin, are sometimes considered to be a warning of more serious incidents to come (17). An analysis of the structure of these two types of tissue should make it possible to determine the physiological and mechanical factors predisposing them to adverse decompression events. The recompression procedures used to treat the symptoms of this minor incidents (which may sometimes be acutely painful) by redissolving the gaseous phase have a high rate of success. This suggests that a mechanical and thermodynamic approach to decompression should effectively describe, at least in part, the sequential events leading to the occurrence of the bends.

Articular symptoms are characterized by sharp localized pain around joints that, if not relieved by recompression, may increase in intensity in the hours following decompression, to reach a peak sometimes after 
several hours. The pain then gradually decreases and disappears. This suggests that there is a slow component driving the dynamics of the gaseous phase responsible for the symptoms.

Articular bends mostly affect the knees and shoulders. Experimental investigations tend to demonstrate the formation of a non negligible volume of separated gas in tendons and ligaments after decompression (35). The disappearance of symptoms after recompression may be only transient, with a reoccurrence of pain in the same area after subsequent decompression. This suggests that a tissular rather then a vascular gaseous phase is responsible for the symptoms. This hypothesis is supported by X-ray observations of joints clearly showing an extravascular free gas phase (17).

The formation of a large number of microbubbles that gradually coalesce and distend the volume within which they are growing may lead to the compression of a terminal nerve zone in stiff tissues such as tendons. Such a mechanism would be expected to cause local pain.

Several studies, including that of Vann (35) focusing on the results of altitude decompressions after a denitrogenation period using pure oxygen, have suggested that joint bends involve tissues in which bubbles form slowly. Indeed, long denitrogenation periods are required to reduce the risk of such incidents markedly (33). This finding is supported by the work of Reeves and Beckman, who tried to establish decompression thresholds after long periods of exposure in dogs (38). Finally, this finding is entirely consistent with the slow, sometimes intermittent flow of blood in the tendons (18).

The hypothesis of a role for the tendons in the bends was formulated some time ago (25). Hills (16) subsequently proposed a biophysical model for simulating this mechanism. The affected zone has an intracellular and an extracellular part, and microbubbles form in the intracellular medium. In this approach, gas exchanges between the cells and the blood are thought to be limited by diffusion. As this structural model is very small, operating at the scale of micrometers, a very low diffusion coefficient had to be introduced $\left(\# 10^{-13} \mathrm{~m}^{2} / \mathrm{s}\right)$. Hills justified this choice by citing the diffusion study on muscle samples carried out by Fenichel and Horowitz (16) and presenting the results of his own experiments on rabbit muscles. However, Kronheim (20) subsequently showed, in studies of the saturation kinetics of rabbit eyes, that such low diffusion values were entirely inconsistent with his results and, therefore highly improbable. The use of such a low coefficient is also incompatible with the results of studies estimating oxygen permeability coefficients at the level of the cell (29).

For simulations of the kinetics of gas exchanges between the blood and a target tissue or between the target tissue and microbubbles within it, a slow diffusion process is often chosen, to ensure the accurate reproduction of the duration of saturation and the timing of decompression incidents. Many models assume the 
presence of low diffusion coefficient zones to reproduce such kinetics. For example, rather than assuming slow gas diffusion in cells, Gernhardt (11) assumed that the microbubbles are surrounded by shells displaying low levels of diffusion, thereby greatly slowing the rate of bubble growth. The diffusion coefficient within these shells was considered to be an order of magnitude greater than suggested by Hills (16) and thus three orders of magnitude lower than the coefficient of the gas in water. The selection of such a low value by Gernhardt remains puzzling, because such shells, if they exist, probably consist of phospholipids and proteins. Nevertheless, Gernhardt's approach is more realistic than that of Hills: low diffusion coefficients throughout the tissue would imply very low growth rates for the bubbles in the target tissue, so that the instantaneous formation of the separated phase when a supersaturation state is reached in this tissue, as advocated by Hills, is, in fact, impossible. Nikolaev (24) also introduced a very low diffusion coefficient into his model of encapsulated bubbles growing between cells. More recently, Payne and Chappell (10), in their model of microbubbles production at capillary endothelial level, also selected a low diffusion coefficient, based on the work and hypothesis of Srinivisan (28).

The introduction of low diffusion coefficients - a puzzling hypothesis for biological media - can be avoided by introducing millimetric diffusion distances (19). Hempleman (14) used this approach in the construction of his articular bends model. Hempleman considered a semi-infinite slab of tissue, within which gas exchanges were limited by diffusion. He considered this slab of tissue to represent a piece of cartilage, within which the bubbles responsible for symptoms can form. Solving this simple diffusion problem, he concluded that the amount of inert gas accumulated in the tissue was proportional to $P \sqrt{t}$ for exposures at a pressure $P$ characterized by durations $t$ of less than $100 \mathrm{~min}$. He then defined a gas excess threshold constituting the decompression criterion. This work provided the foundations for the RNPL 1968 Royal Navy Air Diving Tables. This model, although safe for exposures of moderate duration, proved inadequate for longer periods. However, Hempleman did not model bubble growth in his approach, and this is clearly a major limitation to the application of the model to a broad range of profiles.

The other decompression models used as the basis of calculations in commonly used procedures do not use a specific target tissue to predict the occurrence of decompression sickness. Instead, they consider a wide range of potential target tissues with a large range of saturation half-times $(7,36,37,40)$. They therefore cannot be considered to deal with the decompression problem from a physiological point of view.

We propose here a new biophysical decompression model in which the target tissue, representing a joint, is divided into two neighbouring parts that are perfused at different rates, with exchanges occurring 
between these two parts over distances of a few millimeters. This model is also based on the combination of various published approaches to nucleation and microbubbles growth, making it physically and physiologically consistent. The parameters of the resulting biophysical model have been correlated using COMEX air and heliox database in particular. This database can be used to estimate the risk of articular bends for a wide range of exposures in term of pressure and duration.

\section{Model description}

We assume a representative articular zone within which the formation of bubbles during decompression may cause pain. This zone can be broken down into two parts exchanging gas by perfusion with the blood. These parts will be called Medium I and Medium II. Exchanges by diffusion occur also between Medium I and Medium II (see Fig. 1).

Fig.1.

\subsection{Saturation phase}

Let us consider the first phase of an exposure, consisting of the breathing of a mixture of oxygen and $\mathrm{N}$ inert gases at a given pressure $\mathrm{P}_{\mathrm{amb}}$. During this phase, the inert gases gradually dissolve in the body. In the target tissue, no separated gas phase is present, other than pre-existing gas micronuclei. According to the perfusion hypothesis, the resulting venous inert gas tension of gas $\mathrm{j}$ in this tissue, $P_{v, j}$, can be expressed as follows :

$$
P_{v, j}=\frac{\dot{q_{I}} P_{I, j}+\dot{q_{I I}} P_{I I, j}}{\dot{q}}
$$

with

$\dot{q}=\dot{q}_{I}+\dot{q}$

where :

- $\dot{q}_{I}$ blood flow rate crossing Medium I ( $\left.\mathrm{m}^{3} / \mathrm{s}\right)$

- $\dot{q}_{\text {II }}$ blood flow rate crossing Medium II ( $\left.\mathrm{m}^{3} / \mathrm{s}\right)$ 
- $\dot{q}$ total blood flow rate crossing the articulation zone considered $\left(\mathrm{m}^{3} / \mathrm{s}\right)$

- $\quad P_{I, j}$ inert gas tension in Medium I (Pa)

- $\quad P_{I I, j}$ inert gas tension in Medium II (Pa)

The gas exchanges equations for each gas $\mathrm{j}(\mathrm{j} \in[1 ; \mathrm{N}])$, inspired by the formulations of Hills (16), take into account the perfusion/diffusion hypothesis chosen for the model. The global rate of inert gas exchanges between the articular zone and the blood, expressed in $\mathrm{mol} / \mathrm{s}$, can be written taking into account the perfusion hypothesis selected :

$\dot{q} S_{b, j}\left(P_{a, j}-P_{v, j}\right)=V_{I} S_{I, j} \frac{d P_{I, j}}{d t}+V_{I I} S_{I I, j} \frac{d P_{I I, j}}{d t}$

with :

- $S_{I, j}$ inert gas solubility coefficient in Medium I $\left(\mathrm{mol} / \mathrm{m}^{3} / \mathrm{Pa}\right)$

- $S_{I I, j}$ inert gas solubility coefficient in Medium II $\left(\mathrm{mol} / \mathrm{m}^{3} / \mathrm{Pa}\right)$

- $S_{b, j}$ inert gas solubility coefficient in the blood $\left(\mathrm{mol} / \mathrm{m}^{3} / \mathrm{Pa}\right)$

- $\quad P_{a, j}$ arterial inert gas tension $(\mathrm{Pa})$

- $\quad V_{I}$ Medium I volume $\left(\mathrm{m}^{3}\right)$

- $\quad V_{I I}$ Medium II volume $\left(\mathrm{m}^{3}\right)$

The equations for the amount of substance variations for each medium (each perfused by a given blood flow rate) are similar but take into account the diffusion exchanges with the other medium i.e. for Medium I :

$V_{I} S_{I, j} \frac{d P_{I, j}}{d t}=k_{d, j}\left(P_{I I, j}-P_{I, j}\right)+\dot{q}_{I} S_{b, j}\left(P_{a, j}-P_{I, j}\right)$

and for Medium II :

$V_{I I} S_{I I, j} \frac{d P_{I I, j}}{d t}=k_{d, j}\left(P_{I, j}-P_{I I, j}\right)+\dot{q}_{I I} S_{b, j}\left(P_{a, j}-P_{I I, j}\right)$

with $k_{d, j}$ the equivalent rate of exchange by diffusion between the two media in $\mathrm{mol} / \mathrm{Pa} / \mathrm{min}$. 
The inter-media diffusion exchange rates for each gas may be expressed using two purely geometric coefficients, $\psi_{I}$ and $\psi_{I I}$, reflecting the diffusion distances of each medium and the properties of the gas in the media (solubility, coefficient of diffusion) :

$k_{d_{-} I I, j}=\frac{k_{d, j}}{V_{I I} S_{I I, j}}=\frac{1}{\frac{\psi_{I} S_{I I, j}}{D_{I, j} S_{I, j}}+\frac{\psi_{I I}}{D_{I I, j}}}$

with :

- $D_{I, j}$ inert gas diffusion coefficient in Medium $\mathrm{I}\left(\mathrm{m}^{2} / \mathrm{s}\right)$

- $\quad D_{I I, j}$ inert gas diffusion coefficient in Medium II $\left(\mathrm{m}^{2} / \mathrm{s}\right)$

- $\quad \psi_{I}, \psi_{I I}$ geometric parameters describing the diffusion path between the two media $\left(\mathrm{m}^{2}\right)$

If the media are modelled as two parallel slabs with thicknesses $2 d_{I}$ and $2 d_{I I}$ respectively, it can be shown that

$\psi_{I}=\frac{d_{I} d_{I I}}{2}$

and

$\psi_{I I}=\frac{d_{I I}^{2}}{2}$

The following parameters :

$k_{I, j}=\frac{\dot{q}_{I} S_{b, j}}{V_{I} S_{I, j}}$

and

$k_{I I, j}=\frac{\dot{q_{I I}} S_{b, j}}{V_{I I} S_{I I, j}}$

are the perfusion coefficients characterizing the gas exchanges.

Equations (2.4) and (2.5) closely resemble the Haldane formulation, but have been modified to take into account exchanges by diffusion between media I and II. Equation (2.3) can be deduced by summing equations (2.4) and (2.5), using also equations (2.1) and (2.2). 
If we introduce the biophysical parameters :

$\omega_{j}=\frac{V_{I I} S_{I I, j}}{V_{I} S_{I, j}}$

i.e. the capacity of Medium II over the capacity of Medium I to contain inert gas $\mathrm{j}$ and the flow rate ratios

$a_{I}=\frac{\dot{q_{I}}}{\dot{q}}$

and

$a_{I I}=\frac{\dot{q_{I I}}}{\dot{q}}$

we obtain :

$\left(k_{I, j}+\omega_{j} k_{I I, j}\right)\left(P_{a, j}-a_{I} P_{I, j}-a_{I I} P_{I I, j}\right)=\frac{d P_{I, j}}{d t}+\omega_{j} \frac{d P_{I I, j}}{d t}$

\subsection{Gas exchanges during a subsequent decompression phase}

After this period of exposure, the subject undergoes a decompression. Microbubbles may form from pre-existing micronuclei in the target tissue during the decompression phase. The presence of these microbubbles and changes in their dimensions must be taken into account when describing the kinetics of gas exchanges between the media and blood. This has been theoretically explained by Van Liew (31). It makes the equation system described above more complex.

Nevertheless, we will assume that the microbubbles formed in Medium II are not sufficiently numerous and voluminous at any one point in time to alter gas exchanges between Medium II and the blood. This results in microbubbles dynamics being modelled exclusively for Medium I, which thus constitutes the critical zone of the target tissue (see Fig.1). This would imply that Medium I has a larger initial density of gas micronuclei than Medium II.

The gas exchanges associated with gas $\mathrm{j}$ during the decompression phase may therefore be expressed as follows :

$\dot{q} S_{b, j}\left(P_{a, j}-P_{v, j}\right)=\frac{d n_{I, j}}{d t}+V_{I I} S_{I I, j} \frac{d P_{I I, j}}{d t}$

with 
$n_{I, j}=\left(1-v_{I_{-} b}\right) S_{I, j} P_{I, j} V_{I}+n_{I_{-} b, j}$

where :

- $n_{I, j}$ total number of moles of inert gas in Medium I

- $\quad n_{I_{-} b, j}$ total number of moles of inert gas in the microbubbles population

- $v_{I_{-} b}$ total volume of separated gas per $\mathrm{m}^{3}$ of target tissue

From equation (2.15), using the approximation $v_{I_{-} b}<<1$ :

$\frac{d n_{I, j}}{d t}=\frac{d P_{I, j}}{d t} S_{I, j} V_{I}+\frac{d n_{I_{-} b, j}}{d t}$

Equation (2.5) remains true, because the microbubbles potentially formed in Medium II can be neglected. Note that equation (2.17) is not required if no microbubbles have yet formed in Medium I. We explain below the conditions in which the micronuclei present in Medium I can be recruited and transformed into microbubbles.

Let us suppose, based on the work of Yount (39), that the initial density distribution of micronuclei (number of micronuclei having a radius greater than $r$ per cubic metre before exposure) in Medium I follows an exponential law :

$N_{I}(r)=N_{I_{-} \max } e^{-A_{I} r}$

with :

- $A_{I}$ coefficient of the exponential law $\left(\mathrm{m}^{-1}\right)$

- $N_{I_{-} \text {max }}$ total number of micronuclei per $\mathrm{m}^{3}$ of target tissue initially present in Medium $\mathrm{I}_{\left(\mathrm{m}^{-3}\right)}$

In other words, small micronuclei are much more numerous than large micronuclei. However, the chosen approach, involving the consideration of spherical micronuclei, could be called into question. In other approaches, tissues are thought to contain hydrophobic crevices, such as cells contact zones (24). More complex mechanisms of micronuclei release have been modelled and discussed (30). Modern models (10) aim to represent such possibilities but mostly assume that microbubbles form in the capillary endothelium, within caveolae, for example (6). In the model presented here, we will follow the simplified approach adopted by Yount 
(39), Gernhardt (11) and Van Liew (31), in which micronuclei and microbubbles are considered as spherical elements.

\subsection{Conditions of microbubbles formation}

According to Yount (39), the total density of microbubbles generated $N_{I_{-} \text {tot }}$ at a given time during a decompression is related to the maximum supersaturation level reached up to this time point and, to a certain extent, to the level of compression associated with the exposure, which may eliminate some of the micronuclei by crushing :

$$
N_{I_{-} t o t}=N_{I}\left(r_{0}\right)=N_{I_{-} \max } e^{-A_{I} r_{0}}
$$

with, formulated to take the metabolic gases and water vapour into account :

$$
r_{0}=\frac{\alpha_{I}}{P_{I, s s}-\phi_{I} P_{c r u s h}-\Pi}
$$

where

$$
P_{I, s s}=\sum_{j=1}^{N} P_{I, j}-\left(P_{a m b}-\beta_{I}\right)
$$

and

$$
P_{c r u s h}=\left[\left(P_{a m b}-\beta_{I}\right)-\sum_{j=1}^{N} P_{I, j}\right]_{\max }
$$

$r_{0}$ is the minimal initial radius of the nuclei recruited by the maximum supersaturation level $P_{I, s s}$ following a crushing pressure $P_{c r u s h}$. The formulation of the supersaturation $P_{I, s s}$ is standard but the elastic pressure in Medium I due to the total gas volume formed, $\Pi$, is taken into account in the nuclei recruitment relation above (see next paragraph). $\alpha_{I}(\mathrm{~N} / \mathrm{m})$ and $\phi_{I}$ are the parameters of the nuclei recruitment law. According to Yount (39), these parameters can be linked to the surface tension and to the crumbling compression of a skin in periphery of the nuclei, stabilizing it. 
Note that :

$>$ For a decompression after a saturation state, the crushing pressure corresponds to the oxygen window $(3,16,32)$; this demonstrates the potential effect of oxygen on a micronuclei population.

According to Yount's approach, based on the stabilisation of micronuclei by surface-active compounds until their activation (39), the coefficients of equation (2.20) are related to the surface tension of Medium I:

$\phi_{I}=1-\frac{\alpha_{I}}{2 \gamma_{I}}$

$>$ The density of micronuclei recruited is so all the more important that $r_{0}$ is small i.e. that the supersaturation level is high during the decompression.

During decompression, $r_{0}$ reaches a minimum corresponding to the supersaturation peak in Medium I : $r_{0} \min$. There is an instant associated with this event : $t_{r_{0_{-} \min }}$. Consequently, the maximal density of micronuclei recruited during decompression may be expressed as follows :

$N_{I_{-} \text {tot_max }}=N_{I_{-} \max } e^{-A_{I_{1} r_{-} \min }}$

\subsection{Total volume of microbubbles generated}

Each gas nucleus recruited generates a microbubble according to a growth rate linked to its dimension at a given instant. The total volume (per cubic metre of tissue) of microbubbles generated in Medium I is obtained by summing the micronuclei population involved in the amplification :

$v_{I_{-} b}=\left\{\begin{array}{l}\int_{r_{0}}^{+\infty}-\frac{d N_{I}}{d r} \frac{4 \pi}{3} R_{r}^{3} d r \text { si } t \leq t_{r_{O_{-} \min }} \\ \int_{r_{0_{-} \min }}^{+\infty}-\frac{d N_{I}}{d r} \frac{4 \pi}{3} R_{r}^{3} d r \text { si } t \geq t_{r_{O_{-} \min }}\end{array}\right.$

where $R_{r}$ is the radius (at the instant considered) of a microbubble generated from a nucleus having an initial radius $r$. 
Moreover, assuming that the inert gases concerned can be considered as perfect gases, the total number of moles of gas $\mathrm{j}$ accumulating in the microbubbles may be expressed as follows :

$$
n_{I_{-} b, j}=\left\{\begin{array}{l}
\frac{1}{\mathfrak{R} T} \int_{r_{0}}^{+\infty}-\frac{d N_{I}}{d r} V_{I} P_{b_{r_{-} I, j}} \frac{4 \pi}{3} R_{r}^{3} d r \text { si } t \leq t_{r_{O_{-} \min }} \\
\frac{1}{\mathfrak{R} T} \int_{r_{0_{-} \min }}^{+\infty}-\frac{d N_{I}}{d r} V_{I} P_{b_{r_{-} I, j}} \frac{4 \pi}{3} R_{r}{ }^{3} d r \text { si } t \geq t_{r_{O_{-} \min }}
\end{array}\right.
$$

with $P_{b_{r_{-} I, j}}$ the inert gas partial pressure in the microbubbles generated from a nucleus with an initial radius $\mathrm{r}$.

If we assume that the microbubbles are in a mechanical equilibrium at all time points, then the following relationship applies, linking the different gas pressures in the microbubble of radius $R_{r}$ :

$$
\sum_{j=1}^{N} P_{b_{r_{-} I, j}}+P_{b_{r_{-} O_{2}}}+P_{b_{r_{-}-O_{2}}}=P_{a m b}+\frac{2 \gamma_{I}}{R_{r}}+\Pi-P_{H_{2} O}
$$

where the elastic pressure imposed by Medium I (degree of rigidity) is introduced to limit microbubble growth and maximum size :

$$
\Pi=K v_{I_{-} b}
$$

where $K$ is the elastic bulk modulus in the target tissue in Pa.

We can assume that the metabolic gases in the microbubbles are in equilibrium at all time points, with the corresponding tension value in Medium I i.e. $P_{b_{r_{-} O_{2}}} \approx P_{I_{-} O_{2}}$ and $P_{b_{r_{-}} \mathrm{CO}_{2}} \approx P_{I_{-} \mathrm{CO}_{2}}$. This last hypothesis, considered by Van Liew in his attempts to model microbubbles in the bloodstream (9), is well founded because the carbon dioxide permeability coefficient is very high and oxygen is consumed in the vicinity of the microbubbles. Moreover, in first approximation, we can assume that the oxygen and carbon dioxide tensions in Medium I are equal to the corresponding venous tension values, i.e. $P_{I_{-} \mathrm{O}_{2}} \approx P_{v_{-} \mathrm{O}_{2}}$ and $P_{I_{-} \mathrm{CO}_{2}} \approx P_{v_{-} \mathrm{CO}_{2}}$.

\subsection{Growth and resorption dynamics of microbubbles}

We have reformulated Van Liew's work (31) to simulate the dynamics of the microbubble/tissue interface, taking into account the elastic constraints imposed by the tissue : 
$\frac{d R_{r}}{d t}=\frac{\Re T \sum_{j=1}^{N} D_{I, j} S_{I, j}\left(P_{I, j}-P_{b_{r_{-} I, j}}\left(\frac{1}{R_{r}}+\sqrt{\frac{k_{I, j}}{D_{I, j}}}\right)-\frac{R_{r}}{3} \frac{d P_{a m b}}{d t}\right.}{P_{a m b}+\frac{4 \gamma_{I}}{3 R_{r}}+\Pi-P_{H_{2} 0}}$

$\frac{d P_{b_{r_{-} I, j}}}{d t}=\frac{3 \Re T}{R_{r}} \sum_{j=1}^{N}\left[D_{I, j} S_{I, j}\left(P_{I, j}-P_{b_{r_{-} I, j}}\right)\left(\frac{1}{R_{r}}+\sqrt{\frac{k_{I, j}}{D_{I, j}}}\right)-P_{b_{r_{-} I, j}} \frac{d R_{r}}{d t}\right] \quad \mathrm{j} \in[1 ; \mathrm{N}]$

The square root terms represent the impact of tissue perfusion on the boundary conditions of the diffusion problem in the neighbourhood of the microbubble Their effects are only significant when the microbubble is large, in rather rapid tissues. The boundary conditions have been discussed by Srinivisan (28), comparing Van Liew (34) and Gernhardt (11) approaches of the problem.

\subsection{Model parameters and resolution}

The physical and physiological parameters of the model are given in Table 1 . The equations presented above define a system that cannot be solved analytically. A numerical approach is necessary to solve the problem. The main aim is to determine the total volume of microbubbles generated in Medium I, $v_{I_{-} b}$, at each time point during and after decompression. The ambient pressure profile is an essential input, so the arterial tension of each gas must be determined at each time point :

$$
P_{a, j}=f_{j}\left(P_{a m b}-P_{H_{2} \mathrm{O}}\right) \quad \mathrm{j} €[1 ; \mathrm{N}]
$$

with $f_{j}$ the inert gas fraction of gas $\mathrm{j}$ in the breathing mixture.

Time was converted into a discrete variable in an explicit manner : we assume the variables $P_{I, j}, P_{I I, j}$, $n_{I_{-} b, j}$ and $v_{I_{-} b}$ are known at time $\mathrm{t}$ and we estimate their value, by solving the equation system for time $\mathrm{t}+\Delta \mathrm{t}$. Several known variables are included among the input data : the depth/time segments characterising the exposure, including the stages, if any, with the corresponding breathing gases, as the saturation state of the body initially.

Table 1 
The convergence and stability criteria of this numerical approach are not known a priori, so the time steps chosen for gas exchange kinetics $(\Delta t)$ and for simulating changes in microbubbles volume $(\Delta \mathrm{d})$ must be chosen with care. The second of these time steps must be much smaller than the first, due to the rapid diffusion exchanges between the microbubbles and the tissue. A sensitivity study was carried out to fix these time steps at reasonable values in terms of CPU time $(\Delta \mathrm{t}=5 \mathrm{~s}$ and $\Delta \mathrm{d}=0.01 \mathrm{~s})$.

The numerical problem was solved with MATLAB as a numerical computing environment and programming language.

\subsection{Parameter ranges and fixed parameters}

The surface tension chosen for both media was $\gamma=0.05 \mathrm{~N} / \mathrm{m}$. This surface tension value was also selected and justified by Van Liew (31).

The solubility and diffusion coefficients of helium and nitrogen, measured in water and oil in particular, have been reviewed elsewhere (22). Here we chose to use the values selected by Burkard and Van Liew (8) and reported in Table 2.

Table 2

The ranges of the other physical and physiological parameters considered during the model correlation phase are given in Table 3. The solubility and diffusion coefficients of helium and nitrogen in each medium can be determined from the last line of this table and Table 2, by correctly weighting the fat fraction when determining homogenized values (sum of conductances for the equivalent diffusion coefficients).

Finally, it is assumed in this model that the sum of the metabolic gas tensions and the water vapour pressure in the two media and in the microbubbles are equal, at each time, to the values for the venous side, supposed constant, i.e. $\beta_{I} \approx 132 \mathrm{mmHg} \approx 0.175$ bar.

Table 3

\subsection{Databank used to correlate the model}

We decided to adjust the physical and physiological parameters of the model in order to represent at best the articular bends risk associated with known decompression procedures. The maximum gas volume 
$v_{I_{-} b_{-} \max }$ potentially generated during or after decompression is thought to be linked to the risk of articular bends. This is a key criterion of the model, as in Hennessy and Hempleman's critical volume hypothesis (15). A relationship linking the risk $r_{D C S}$ and $v_{I_{-} b_{-} \max }$ has to be extracted. This relationship can then been used to estimate the risk for any decompression procedure after any type of exposure, however unusual, including deep, short dives with gas mixtures.

For correlation analysis, we used the risk analysis of the 1986 COMEX tables. It includes tens of thousand of exposures with articular bends as the main decompression sickness cases (mainly located at knee level). As proposed by Shields, in an analysis of North Sea decompression procedures (27), a PrT parameter (the product of $\mathrm{P}$, the exposure pressure in atmospheres, and $\mathrm{rT}$, the square root of the duration of exposure in minutes) was used to evaluate the risk of decompression sickness. It has been established that this risk increases with PrT, as follows:

$r_{D C S}=4.07(\operatorname{Pr} T)^{4.14}$

Based on an analysis of the 1986 COMEX Helium tables, the risk in divers using helium is only half that in divers using air, for a same PrT value.

Another aim of this model was to identify a criterion estimating the risk of an incident during and after decompression following a state of saturation. Again, we used the COMEX database. We considered heliox exposures and the associated COMEX decompression procedures : initial saturation state with $\mathrm{P}_{\mathrm{O} 2}=0.4$ ATA, then decompression at an ascent rate of $50 \mathrm{~min} / \mathrm{m}$ with $\mathrm{P}_{\mathrm{O} 2}=0.5$ ATA up to a 2.5 ATA ambient pressure and, finally, a constant inspired oxygen fraction $F I_{\mathrm{O}_{2}}=0.21$ up to the surface to limit oxygen hazard, with an ascent rate of $60 \mathrm{~min} / \mathrm{m}$. Following a $180 \mathrm{~m}$ saturation exposure, the risk of articular bends is known to be below $1 \%$ (not published). For air saturation exposures, at acceptable nitrogen partial pressures in terms of inert gas narcosis, a similar procedure can be used, but with an ascent rate of more than $100 \mathrm{~min} / \mathrm{m}$. However, the risk is less well known.

We used also the heliox experimental results of Barnard (1) as a reference for adjustment of the parameters of the model. The aim of Barnard's study was to identify decompressions that were acceptable in terms of the bends risk. Human subjects were decompressed from a saturation state, characterized by a pressure level $\mathrm{P}_{1}$ (in ATA), to another pressure level $\mathrm{P}_{2}$ (in ATA). It was shown that, in normoxic breathing conditions, 
there is a linear relationship between these two pressure levels. This relationship was discussed and used by Hennessy and Hempleman (15) in their justification of the critical volume hypothesis :

$P_{1}=1.40 P_{2}+0.57$

The bends risk is not precisely known, as the numbers of subjects and exposures tested were not particularly high. However, it seems likely that this risk is non negligible.

Hennessy and Hempleman (15) derived a similar relationship for air exposures, from a simple model correlated with empirical results. They compared this relationship to the results of Workman's approach (37) :

$P_{1}=1.38 P_{2}+0.52$

These last two relationships constitute information potentially useful for the estimation of model parameters.

Articular bends may sometimes occur during an isobaric breathing gas switch from nitrogen to helium. As a reference, we considered the case of a bends in a knee at 4 ATA (35). It has been suggested that gas counterdiffusion phenomena may account for decompression sickness following such switches (21). The model proposed here should be able to predict this possibility.

Finally, we also used rapid altitude ascent data, with thresholds between 25,000 feet and 38,000 feet to adjust the model. We used the incident rate of $13 \%$ given by Van Liew (33) as the reference value for rapid ascents at $21 \mathrm{kPa}$ without pre-oxygenation (about 2000 subjects).

\subsection{Correlation method}

A long correlation phase is necessary to adjust the model parameters. The principle consists in varying one specific parameter while fixing the others to a preselected value. For each value of the investigated parameters, several groups of simulations are conducted :

- 141 air, air/oxygen and heliox dives following the MT92 procedures (1992 French Labour Ministry tables) in accordance with COMEX 1986 tables

- a heliox saturation decompression from $180 \mathrm{~m}$ 
- the $P_{1}=a P_{2}+b$ pressure echelons proposed by Barnard and Workman for heliox and air

- the air $\rightarrow$ heliox gas switch case at 4ATA

- a rapid ascent at $21 \mathrm{kPa}$ from atmospheric pressure conditions

A relationship between the risk $r_{D C S}$ and $v_{I_{-} b_{-} \max }$ is first searched for the 141 profiles, with a coefficient of determination $\mathrm{R}^{2}$ expected to be above 0.8 . If such a relationship exists, then $v_{I_{-} b} b_{-}$max and the associated risk are determined for the other exposures mentioned above. The obtained risk is then analyzed to measure how true to life it is. After this phase, a new value of the investigated parameter is chosen in its selected range (Table 3). The same group of simulations is carried out in order to verify if an improvement of the correlation appears. This method is repeated until the best value for the parameter is found. Then a new parameter is investigated and so on.

Preferably, a stochastic estimator could be used to correlate the model.

\section{Results}

The optimization phase described in the previous paragraph allowed the selection of two different sets of parameters giving similar results.

For Set A, as shown in Table 4, the half-times $\mathrm{T}_{1 / 2}=\ln 2 / \mathrm{k}$ corresponding to the perfusion coefficients were $690 \mathrm{~min}$ for Medium I and $395 \mathrm{~min}$ for Medium II for nitrogen, $690 \mathrm{~min}$ for Medium I and $280 \mathrm{~min}$ for Medium II for helium. These values suggest that the articular target tissue should be considered a "slow" tissue, not necessarily with a high fat content, when decompression induces local pain.

Such half-times have been considered for 1) saturation and altitude ascent decompressions 2) counterdiffusion analysis 3) rest periods in dry conditions in general. However, for immersed exposures like air and helium bounce dives of short or moderate duration (saturation state not reached), these half-times must be divided by a factor. This factor differs for the bottom period and for the decompression period, in accordance with the different levels of activity of the subject during the exposure (gas exchanges enhancement). Correlation analysis showed that the factors best fitting the data were 8 for the bottom period and 3 for decompression. This implies that, in the worst-case scenario, resulting in decompression sickness, the rate of tissue saturation is higher than that of tissue desaturation. Table 5 presents the half-times best fitting the model.

Table 4 
The correlation analysis tends to suggest that Medium I is likely to be a watery tissue, whereas Medium II is likely to be a tissue with low or moderate fat levels..

Table 5

The relative capacities of the two media to accumulate inert gases (between 4 and 8 for nitrogen and helium) suggests that Medium II volume, in which microbubbles formation is not considered, is larger than Medium I volume. The painful bubbles therefore seem to be restricted to a small part of the articular zone modelled here. Moreover, the parameters $\psi_{I}$ and $\psi_{I I}$, characterizing the pathway of diffusion between Medium I and Medium II, correspond to diffusion distances of 5 to $10 \mathrm{~mm}$. The diffusion gas exchange rate between the two media, for the two inert gases and from Medium II point of view, suggests moderate exchange speeds, neither rapid nor slow : $\mathrm{T}_{1 / 2}$ around $25 \mathrm{~min}$ for helium and around $100 \mathrm{~min}$ for nitrogen, difference in speed inducing the counter-diffusion phenomenon that may cause articular bends. Medium II saturating more rapidly than Medium I, the inter-media diffusion exchange is fundamental for the gas feeding of the microbubbles formed in Medium I during the decompression. Medium II plays a large role on their growth. Moreover, from Medium I point of view, the diffusion gas exchange rate between the two media are rather high for both gases due to the relative capacity of these two media to contain the inert gases. Consequently, while the inter-media diffusion plays a moderate role on the Medium II desaturation rate, it has a major role on Medium I desaturation rate and instantaneous gas content.

It should be noted that the diffusion coefficients used in this model remain in the physical range : it is not necessary to introduce the very low values frequently selected in previous models. Indeed, the model can reproduce the slow bubbles formation suggested by the delayed onset of pain after decompression. There is also no need to introduce small micrometric diffusion barriers with a very low diffusion coefficient around bubbles, as suggested by Gernhardt (11), or to assume poor diffusion within tissues, as proposed by Hills (16) and Nikolaev (24). This model simply considers diffusion paths over several millimetres, as proposed by Hempleman (14).

For practical purposes, and because the results are similar for Set A and Set B, we describe below only the results for Set A. 


\subsection{Open-sea air and heliox dives}

Fig. 2 presents the $v_{I_{-} b_{-} \max }$ results after correlation for the 141 air, air/oxygen et heliox dives simulated according to MT92 procedures (1992 French Labour Ministry tables), the PrT value for each profile being translated into a risk $r_{D C S}$, as suggested by COMEX analysis (see relation 2.32). The main objective of the correlation analysis carried out on model parameters was to identify a relationship between $r_{D C S}$ and $v_{I_{-} b} b_{-} \max$. A logarithmic relationship seems to fit the data well, given the broad diversity of exposures considered. A volume close to 0.025 implies a risk close to $1 \%$, whereas a volume close to 0.007 implies a risk close to $0.01 \%$. Thus, the target tissue is highly sensitive above a potential separated gas volume $v_{I_{-} b \text { max }}$ close to 0.02 . The adjective "potential" is appropriate because the risk reflects both :

\footnotetext{
* the probability of a joint tissue having the configuration studied, with more rapid saturation than desaturation and slow exchange rates for the desaturation phase

* the probability of pain being generated at a given separated volume
}

In this context, it is easy to account for the commonly observed inter-individual and intra-individual variability in the risk of decompression sickness.

Fig.2.

Fig. 3 and Fig.4 illustrate the behaviour of the model for two simulated dives : $12 \mathrm{~m} / 360 \mathrm{~min}$ air and 60 $\mathrm{m} / 50 \mathrm{~min}$ heliox, with breathing pure oxygen at the $6 \mathrm{~m}$ stage for this latter. They show the ambient pressure, changes in inert gas tensions in both media and the volume of gas generated in Medium I. It appears that 1) there is a rapid clamping phenomenon in Medium I, the microbubbles being formed rapidly by absorption of the gas dissolved in their neighbourhood, resulting in an equilibrium between the dissolved gas in Medium I and the partial pressure of nitrogen in the microbubbles being reached within a few seconds or minutes 2/ Medium II feeds in gas the microbubbles formed in Medium I, through diffusion between the two media, over a period of more than two hours for air dives, consistent with the delays sometimes observed in the onset of bends symptoms. Fig. 5 shows the changes in the radius of gas micronuclei activated during decompression for the 
heliox dive considered (minimum radius $r_{0_{-} \min }=2 \mu \mathrm{m}$ ). Breathing oxygen at $6 \mathrm{~m}$ seems to inverse the radius evolution but cannot prevent the final expansion of the bubbles when surfacing. This decompression stage therefore appears to be only moderately efficient.

Fig.3.

Fig.4.

Fig.5.

\subsection{Saturation exposures}

Concerning the decompression procedure considered for saturation heliox exposure $(50 \mathrm{~min} / \mathrm{m}$ at $\mathrm{P}_{02}=0.5$ ATA up to a 2.5 ATA ambient pressure then $F I_{O_{2}}=0.21$ with a $60 \mathrm{~min} / \mathrm{m}$ ascent rate), a volume $v_{I_{-} b_{-} \max }=0.026$ was obtained when surfacing (Fig.6), giving a risk $r_{D C S} \approx 0.8 \%$, with a higher risk close to the surface, as observed in practice. We also checked that decompression from deeper levels, such as $300 \mathrm{~m}$, results in a risk lower than $5 \%\left(r_{D C S} \approx 1.8 \%\right)$, as observed in practice too. COMEX indeed demonstrated that such a procedure can work at depths of more than $180 \mathrm{~m}$, but with a slightly higher risk of decompression sickness.

A similar decompression procedure after air saturation exposure $\left(100 \mathrm{~min} / \mathrm{m}\right.$ at $\mathrm{P}_{02}=0.5$ ATA up to a 2.5 ATA ambient pressure then $F I_{O_{2}}=0.21$ with a $120 \mathrm{~min} / \mathrm{m}$ ascent rate) has been tested for shallow depths $(<4$ ATA to limit narcosis events). A risk close to $0.02 \%$ was obtained for Set A ( $0.1 \%$ for Set B), apparently lower than the risk of the heliox procedure for high pressure levels. Nevertheless, ascent rates of less than $75 \mathrm{~min} / \mathrm{m}$ are associated with a relatively high risk of decompression sickness $(>0.5 \%)$, particularly if Medium II contains lipids (Set B).

Fig.6.

\section{3. $\mathrm{P}_{1}=\mathrm{a}+\mathrm{bP} \mathrm{P}_{2}$ relationships}

An essential correlative element is the demonstration that the decompression model can approach the linear relationships found by Barnard and Workman for heliox (normoxic) and air, respectively. Hills (16), Hennessy and Hempleman (15) and Yount (40) used these relationships to correlate and validate their models. 
We tested such pressure steps from saturation levels and found the following linear relationships (with a surprising low level of dispersion, $\mathrm{R}^{2}=0.99$ ), after correlation :

$$
P_{1}=1.32 P_{2}+0.66 \quad \text { for heliox }
$$

and

$$
P_{1}=1.50 P_{2}+0.40 \quad \text { for air }
$$

These results compare favourably with previously reported findings (in terms of curve shape and coefficients). The corresponding $v_{I_{-} b_{-} \max }$ volumes for heliox and air are 0.018 and 0.012 , respectively, with corresponding risks of $r_{D C S}=0.2 \%$ and $0.05 \%$. Barnard's experiments, including only a moderate number of subjects, probably nonetheless generated a high level of risk when the procedure was considered successful. Moreover, Workman's linear relationship, deduced from his M-values theory for the slowest tissue, is probably safer than Barnard's proposition, because his model was validated on a large range of exposures, including some of long duration. So, a factor-four difference between the two decompression sickness rates is not particularly surprising, but the absolute values nevertheless appear to be low.

\subsection{Rapid ascents to altitude}

After correlation, the model showed that, for hypobaric incursions down to $21 \mathrm{kPa}$, we obtain a volume $v_{I_{-} b_{-} \max }=0.018$, giving a risk of $0.2 \%$. This is far lower than the $13 \%$ risk extracted from the Van Liew database (33).

This result is illustrated in Fig.7, which shows the ambient pressure, changes in inert gas tensions in both media and the volume of gas generated in Medium I during a $2 \mathrm{~h}$ exposure at $21 \mathrm{kPa}$ followed by a rapid return to 1 ATA over a period of 5 minutes. Gas volume peaks about $100 \mathrm{~min}$ after the start of the ascent, but the volume decrease is very slow for prolonged exposure, consistent with the observation that bends may occur several hours after the ascent (33).

Fig.7. 


\subsection{Counterdiffusion events}

We tried to estimate the probability of occurrence of articular bends described by Vann and Thalmann (35), following an air to heliox switch at 4 ATA. It has been demonstrated that a supersaturation state does indeed occur in Medium I. This can be explained by the helium being taken up more rapidly than nitrogen is released, in medium I (difference in the rate of exchange by diffusion between the two media for the two gases). Nevertheless, the total volume of the bubbles generated is small, giving a risk between $0.005 \%$ and $0.04 \%$, with the highest value obtained if Medium II contains lipids (Set B of parameters).

\section{Discussion}

It is interesting to analyse the two set of parameters selected after the correlation phase. First, a large population of micronuclei must be selected : the clamping phenomena in Medium I requires high microbubbles densities (31). However, increasing or decreasing the $N_{I_{-} \max }$ value chosen by a factor 10 also gave good correlation results, so this parameter is not critical. Only small densities $\left(<100\right.$ micronuclei $\left./ \mathrm{cm}^{3}\right)$ are clearly incompatible with the results. Moreover, the selected $A_{I}$ value (coefficient associated with the exponential law describing the initial micronuclei dimensions distribution) suggests that a similar number of microbubbles are recruited during the different decompressions simulated. In other words, a large and similar pool of micronuclei ( $>1 \%$ of the total pool) is always involved in conditions of supersaturation in Medium I. It is the saturation level of Medium II — the inert gas reservoir - during decompression that controls the final volume of gas generated, rather than the distribution of micronuclei as suggested by Yount's approach in his VPM model (40). The crushing phenomenon can also be ignored $\left(\alpha_{I}=0.1 \mathrm{~N} / \mathrm{m}\right.$ for $\left.\gamma_{I}=0.05 \mathrm{~N} / \mathrm{m}\right)$ without impairing the fit to empirical data, and this, again, is not consistent with Yount's model.

A high value (>1 MPa) was selected for the elastic bulk modulus, consistent with the target tissue being rather stiff. Such elastic properties may be found in tendons and ligaments. This implies a strong contribution of the elastic forces to the internal pressure of the microbubbles during total volume increase. Fig. 3 illustrates this finding for changes in $P_{I, N_{2}}$ : the gas tension, reflecting the nitrogen pressure in the microbubbles, rapidly decreases towards ambient pressure (clamping) but then gradually increases to reach a maximum imposed by the elastic forces. This maximum corresponds to a transient tension equilibrium between the two media, with 
Medium II no longer supplying Medium I with inert gas, thus resulting in the cessation of microbubbles growth. The large elastic deformation pressure found to fit with the risk criterion $(>200 \mathrm{mmHg})$ is not consistent with the $10 \mathrm{mmHg}$ value used by Hills (16) and also adopted by Hennessy and Hempleman (15). This implies that nerve ending may be less sensitive than previously thought to increases in internal pressure in the tendons or ligaments.

The difference in the initial micronuclei densities assumed for Medium I and Medium II is consistent with the nature proposed for Medium I : the strong mechanical stress levels occuring inside tendons predispose them to the production of a large micronuclei populations compared to the neighboring parts represented by Medium II.

Concerning the distances between the two media, the $5 \mathrm{~mm}$ to $10 \mathrm{~mm}$ values selected are realistic if we take joint dimensions into account. This is an important element of the correlation, allowing to keep physically realistic values of the diffusion coefficients (magnitude $10^{-9} \mathrm{~m}^{2} / \mathrm{s}$ ), for both nitrogen and helium. It may account for the slow rate of microbubbles growth without the need to introduce for media very low rates of diffusion, as proposed by Hills (16) and Gernhardt (11).

Another interesting result concerns the distribution of lipids between the two media : Medium I seems to be a watery part of the joint (corresponding to the nature of tendons), while Medium II may be watery or contain low to moderate amounts of fat. Joints, such as the knee in particular, may contain lipid pads. The correlation analysis also showed that the target tissue zone (Medium I) was smaller than its gas reservoir (Medium II), consistent with exchanges between a tendon and the entire volume of the joint.

The long perfusion half-times selected for Medium I and Medium II, to fit the empirical data, particularly for the desaturation phase, suggest that such a conformation of blood circulation is probably rare, with this probability contributing to the final risk.

There may be a simple, logical reason for the low risk value obtained for the linear $\mathrm{P}_{1}=\mathrm{a}+\mathrm{bP} \mathrm{P}_{2}$ relationships and for the counterdiffusion case analysed. Indeed, in these situations, slow desaturation rate conformations have a given level of probability, as for open-sea dives. But starting from a saturation state maximises the risk, because the rapid saturation kinetics proposed by the model is not necessary. A factor greater than one must be applied to the two risks found here to take this into account. Comparison of the $13 \%$ risk value obtained for rapid ascents at $21 \mathrm{kPa}$ with the estimate of $0.2 \%$ in this study suggests that such a multiplication factor must be applied to the estimated risk to obtain a realistic value when the decompression starts from a saturated state of the body. In other words, the worst configuration (slow perfusion levels, large micronuclei 
population, high elastic bulk modulus...) for preventing the occurrence of bends considered here certainly involves a rapid rate of saturation of the target tissue. Such saturation characteristic is nevertheless rare, accounting for the much higher risk that must be attributed to incursions or gas switches from saturation states. Applying such a rule gives a higher risk of decompression sickness for the Barnard and Workman linear relationships, respectively, which is probably close to reality. For the counterdiffusion simulation, this risk is also presumably higher, making such problems a real possibility, as observed in practice. This is particularly true for knees, which have a relatively high lipid content.

\section{Conclusion}

The decompression model proposed here overcomes the pitfalls of previous approaches, which were not sufficiently physiological or were based on questionable physical hypothesis, such as very low diffusion coefficients. A target joint (e.g. knee, shoulder,...) is split into two parts exchanging with the blood by perfusion and with each other by diffusion over distances of several millimetres. Exchanges of the gases commonly used in breathing mixtures for diving (nitrogen, helium), and the formation, amplification and resorption of microbubbles during and after decompression were simulated around a critical gas volume criterion, to estimate the probability of bends occurring. Two sets of parameters gave the best fit to a broad range of empirical results for the risk of articular bends. The physical properties (solubility, diffusion coefficients) were considered for both the inert gases. The correlation phase showed that: 1) the medium within which profuse microbubbles formation occurs is watery, consistent with the tendons being potential target sites; 2) a clamping phenomenon occurs at this site from the beginning of the decompression, greatly slowing gas exchanges, so that the oxygen window constitutes a major driving force for inert gas elimination; 3) the total microbubbles volume potentially capable of inducing pain (nerve compression due to tissue deformation) generates a non negligible overpressure; 4) there is a simple relationship linking a total separated gas volume and a bends risk, this risk increasing exponentially with increasing volume; 5 / for the worst physiological configuration considered, the two media may become saturated at a moderate rate if exercise is practised during exposure, whereas desaturation rates are very slow; 6) the risk associated with a given procedure takes into account the intrinsic probability of the worst physiological configuration and the occurrence of pain for a given gas volume; 7) the medium in contact with the medium in which the bubbles form may be watery or contain moderate levels of fat, consistent with the nature of the joint tissues surrounding tendons and 8) the delayed occurrence of bends may be accounted for by the total 
gas volume kinetics, with a progressive effect due to the slow provision of gas by intertissue diffusion from the gas reservoir (Medium II).

This model may be considered descriptive in terms of decompression mechanisms. The slow rate of gas exchanges during decompression, inducing the marked expansion of microbubbles, demonstrates that the duration of decompression stages must be greatly increased to decrease decompression sickness risk markedly. This risk results mostly from the gas load before decompression. It is therefore not possible to develop short decompression procedures for deep dives : very long decompressions are always required. Furthermore, in the history of decompression models, successive models have added increasingly long half-times for their slowest compartments, to decrease the bends risk of long/deep exposures, resulting in longer decompression periods. The description of the decompression mechanism suggested here may account for this. Given the oxygen window effect on gas egress, enriched oxygen mixtures or pure oxygen should be used to minimise the risk of bends during and after decompression. 


\section{References}

1. Barnard, E.E.P., 1976. Fundamental studies in decompression from steady-state exposures. Proceedings of the Fifth Symposium on Underwater Physiology, ed CJ Lambertsen, Bethesda, Maryland, Federation of American Societies for Experimental Biology.

2. Bert, P., 1878. La pression barométrique. Masson, Paris.

3. Behnke, A.R., 1967. The isobaric (oxygen window) principle of decompression. In: The New Thrust Seaward. Trans. $3^{\text {rd }}$ Annual Conf. Marine Tech. Soc., San Diego, Washington, DC : Marine Technology Society .

4. Blatteau, J.E., Hugon M., Gardette B., 2008. Deep stops during decompression from 50 to $100 \mathrm{msw}$ didn't reduce bubble formation. UHMS Workshop «decompression and the deep stops » 24-25 June 2008

5. Boycott, A.E., Damant, G.C.C., Haldane, J.S., 1908. The prevention of compressed-air illness. J. Hyg. 8: 342-443.

6. Brubakk, A.O., 2004. Endothelium and bubble injury: the role of endothelium in decompression illness. In: Grandjean B, Meliet JL, eds. $30^{\text {th }}$ Annual Scientific Meeting of the European Underwater Baromedical Society, Ajaccio, Corsica, France, 17-22.

7. Bühlmann, A.A., 1984. Decompression - Decompression sickness. Springer Verlag.

8. Burkard, M.E., Van Liew, H.D., 1994. Simulation of exchanges of multiple gases in bubbles in the body. Resp. Physiol. 95: 131-145.

9. Burkard, M.E., Van Liew, H.D., 1994. Oxygen transport to tissue by persistent bubbles: theory and simulations. J. Appl. Physiol. 77(6): 2874-2878.

10. Chappell, M.A., Payne, S.J., 2006. A physiological model of the release of gas bubbles from crevices under decompression. Respir. Physiol. Neurobiol. 153: 166-180.

11. Gernhardt, M.L., 1991. Development and evaluation of a decompression stress index based on tissue bubble dynamics. PhD dissertation. Philadelphia: Univ. of Pennsylvania Press.

12. Harvey, E.N., 1945. Decompression sickness and bubble formation in blood and tissues. Bulletin of The New York Academy of Medicine 21: 505-536.

13. Harvey, E.N.., Barnes, D.K., McElroy, W.D., Whiteley, A.H., Pease, D.C., Cooper, K.W., 1944. Bubble formation in animals : I. Physical factors. J. Cell. Comp. Physiol. 24(1): 1-22. 
14. Hempleman, H.V., 1952. Investigation into the decompression tables : a new theoretical basis for the calculation of decompression tables. Royal Naval Personnel Research Committee, Report III - Part A, UPS131, Medical Research Council, London.

15. Hennessy, T.R., Hempleman, H.V.,1977. An examination of the critical released gas volume concept in decompression sickness. Proceedings of the Royal Society of London, B197: 299-313.

16. Hills, B.A., 1966. A thermodynamic and kinetic approach to decompression sickness. Thesis, Adelaïde, Libraries Board of South Australia.

17. Hills, B.A., 1977. Decompression sickness - Volume 1: the biophysical basis of prevention and treatment. John Wiley \& Sons.

18. Hills, B.A., 1979. Intermittent flow in tendon capillary bundles. J. Appl. Physiol. 46: 696-702.

19. Homer, L.D., Weathersby, P.K., 1986. How well mixed is inert gas in tissues? J. Appl. Physiol. 60(6): 2079-2088.

20. Kronheim, S., 1976. Inert gas exchange and bubble formation and resolution in the eye. $\mathrm{PhD}$ dissertation. Philadelphia: Univ. of Pennsylvania Press.

21. Lambertsen, C.J., Idicula, J., 1975. A new gas lesion syndrome in man, induced by "isobaric gas counterdiffusion” J. Appl. Physiol. 39(3): 434-443.

22. Lango, T., Morland, T., Brubakk, A.O., 1996. Diffusion coefficients and solubility coefficients for gases in biological fluids and tissues : a review. Undersea Hyperb. Med. 23(4): 247-272

23. Marroni, A., Bennett P.B., Cronje F.J., Cali-Corleo R., Germonpre P., Pieri M., Bonuccelli C., Balestra C., 2004. A deep stop during decompression from 82 fsw (25m) significantly reduces bubbles and fast tissue gas tensions, Undersea Hyperd. Med. , 31: 233-243.

24. Nikolaev, V.P., 2000. Effects of heterogeneous structure and diffusion permeability of body tissues on decompression gas bubble dynamics. Aviat. Space Environ. Med. 71: 723-729.

25. Nims, L.F., 1951. Environmental factors affecting decompression sickness. In : Decompression Sickness, Ed. J.F. Fulton, Saunders, Philadelphia, chapter 8.

26. Pol, B., Watelle, T.J.J., 1854. Mémoire sur les effets de la compression de l'air, appliquées au creusement des puits à houille. Ann. Hyg. Publ. $2^{\text {nd }}$ Ser., 1: 241-279.

27. Shields, T.G., Duff, P.M., Lee, W.B., 1987. Decompression sickness in commercial air dives . $13^{\text {th }}$ Annual Meeting of the Euopean Undersea Biomedical Society, 35-38. 
28. Srinivisan, R.S., Gerth, W.A., Powell, M.R., 1999. Mathematical models of diffusion-limited gas bubble dynamics in tissue. J. Appl. Physiol. 86(2): 732-741.

29. Subczynski, W.K., Hopwood, L.E., Hyde, J.S., 1992. Is the mammalian cell plasma membrane a barrier to oxygen transport? J. Gen. Physiol. 100(1): 69-87.

30. Tikuisis, P., 1986. Modeling the observations of in vivo bubble formation with hydrophobic crevices. Undersea Biomed. Res. 13(2): 165-180.

31. Van Liew, H.D., Burkard, M.E., 1993. Density of decompression bubbles and competition for gas between bubbles, tissue and blood. J. Appl. Physiol. 75(5): 2293-2301.

32. Van Liew, H.D., Conkin, J., Burkard M.E., 1993. The oxygen window and decompression bubbles: estimates and significance. Aviat. Space Environ. Med. 64 : 859-865.

33. Van Liew, H.D., Conkin, J., Burkard, M.E., 1994. Probabilistic model of altitude decompression sickness based on mechanistic premises. J. Appl. Physiol. 76(6): 2726-2734.

34. Van Liew, H.D., Hlastala, M.P., 1969. Influence of bubble size and blood perfusion on absorption of gas bubbles in tissues. Resp. Physiol. 7: 111-121.

35. Vann, R.D., Thalmann, E.D., 1993. Decompression physiology and practice. In : The Physiology and Medicine of Diving, $4^{\text {th }}$ edition, Ed. P. Bennett \& D. Elliott, 376-432.

36. Wienke, B.R., 1990. Reduced gradient bubble model. Int. J. Biomed. Comput. 26: 237-256.

37. Workman, R.D., 1965. Calculation of decompression schedules for nitrogen-oxygen and heliumoxygen dives. US Navy Experimental Diving Unit, Report 6-65, Washington Navy Yard, Washington, DC.

38. Workman, R.D., Bornmann, R.C., 1975. Decompression theory : American practice. In : The Physiology and Medicine of Diving, $2^{\text {nd }}$ edition, Ed. P. Bennett \& D. Elliott, 307-330.

39. Yount, D.E., 1979. Skins of varying permeability: a stabilization mechanism for gas cavitation nuclei. J. Acoust. Soc. Am. 65(6): 1429-1439.

40. Yount, D.E., Hoffman, D.C., 1986. On the use of a bubble formation model to calculate diving tables. Aviat. Space Environ. Med. 57: 149-156. 


\section{Figures}

Fig.1. Diagram of the articular bends biophysical model, showing the two perfused media involved.

Fig.2. Linear relationship linking the risk of decompression sickness occurrence $r_{D C S}$ and the maximum volume of gas formed in the target tissue $v_{I_{-} b_{-} \text {max }}$ corresponding to the simulation of 141 COMEX MT92 dives with Set A parameters

Fig.3. Changes in nitrogen tensions and the volume of gas generated in the target tissue after a $12 \mathrm{~m} / 360$ min air exposure followed by a decompression according to MT92 $-r_{0_{-} \min }=1.8 \mu \mathrm{m}, N_{I_{-} \text {tot }} / N_{I_{-} \max }=16 \%$,

$$
v_{I_{-} b_{-} \max }=0.015 \text {. }
$$

Fig.4. Changes in gas tensions and the volume of gas generated in the target tissue after a $60 \mathrm{~m} / 50$ min heliox exposure followed by a decompression, according to MT92 - $r_{0_{-} \min }=2.0 \mu \mathrm{m}, N_{I_{-} t o t} / N_{I_{-} \max }=13 \%$,

$$
v_{I_{-} b_{-} \text {max }}=0.022 \text {. }
$$

Fig.5. Changes in microbubbles radius in the target tissue after a $60 \mathrm{~m} / 50 \mathrm{~min}$ heliox exposure followed by a decompression according to MT92.

Fig.6. Total volume of gas generated in the target tissue during a linear decompression following a heliox saturation exposure at 19 ATA, according to 1992 French Labour Ministry procedures.

Fig.7. Changes in nitrogen tensions and in the volume of gas generated in the target tissue after a rapid ascent to $21 \mathrm{kPa}$ while breathing air. 


\section{Definition of symbols}

$P_{a m b}$ ambient pressure, $\mathrm{Pa}$

$P_{\text {crush }}$ crushing pressure, $\mathrm{Pa}$

$\mathrm{N}$ number of inert gases considered during the exposure and the associated decompression $\mathrm{j}$ index associated with the gas $\mathrm{j}$ considered, $\mathrm{j} €[1 ; \mathrm{N}]$

$f_{j}$, inert gas fraction of gas $\mathrm{j}$ in the breathing mixture

$F I_{\mathrm{O}_{2}}$, oxygen fraction in the breathing mixture

$P_{a, j}$ arterial inert gas tension, $\mathrm{Pa}$

$P_{v, j}$ venous inert gas tension, $\mathrm{Pa}$

$P_{I, j}$ inert gas tension in Medium I, Pa

$P_{I I, j}$ inert gas tension in Medium II, Pa

$S_{I, j}$ inert gas solubility coefficient in Medium I, $\mathrm{mol} / \mathrm{m}^{3} / \mathrm{Pa}$

$S_{I I, j}$ inert gas solubility coefficient in Medium II, $\mathrm{mol} / \mathrm{m}^{3} / \mathrm{Pa}$

$S_{b, j}$ inert gas solubility coefficient in the blood, $\mathrm{mol} / \mathrm{m}^{3} / \mathrm{Pa}$

$D_{I, j}$ inert gas diffusion coefficient in Medium I, $\mathrm{m}^{2} / \mathrm{s}$

$D_{I I, j}$ inert gas diffusion coefficient in Medium II, $\mathrm{m}^{2} / \mathrm{s}$

$k_{d, j}$ equivalent rate of exchange by diffusion between the two media, $\mathrm{mol} / \mathrm{Pa} / \mathrm{min}$

$k_{d_{-} I I, j}$ rate of exchange by diffusion between the two media associated to Medium II, $\min ^{-1}$

$k_{I, j}$ rate of exchange by perfusion between Medium I and the blood, $\min ^{-1}$

$k_{I I, j}$ rate of exchange by perfusion between Medium II and the blood, $\min ^{-1}$

$\omega_{j}$ relative inert gas content capacity between Medium II and Medium I

$n_{I, j}$ inert gas, total number of moles in Medium I, mol

$n_{I_{-} b, j}$ inert gas, total number of moles in the microbubbles population, mol 
$P_{b r_{-} I, j}$ inert gas partial pressure in the microbubbles generated from a nucleus with an initial radius $\mathrm{r}, \mathrm{Pa}$

$P_{b r_{-} 0_{2}}$ oxygen partial pressure in the microbubbles generated from a nucleus with an initial radius $\mathrm{r}, \mathrm{Pa}$

$P_{I_{-} 0_{2}}$ oxygen tension in Medium I, Pa

$P_{v_{-} 0_{2}}$ oxygen venous tension, $\mathrm{Pa}$

$P_{b_{r_{-} \mathrm{CO}_{2}}}$ carbon dioxide partial pressure in the microbubbles generated from a nucleus with an initial radius

$r, \mathrm{~Pa}$

$P_{I_{-} \mathrm{C0}_{2}}$ carbon dioxide tension in Medium I, Pa

$P_{v_{-} \mathrm{CO}_{2}}$ carbon dioxide venous tension, $\mathrm{Pa}$

$P_{\mathrm{H}_{2} \mathrm{O}}$ water vapour tension at $37^{\circ} \mathrm{C}$, saturation state, $\mathrm{Pa}$

$P_{I, s s}$ supersaturation level in Medium I, Pa

$\psi_{I}, \psi_{I I}$ geometric parameters describing the diffusion path between the two media, $\mathrm{m}^{2}$

$\dot{q}_{\text {I }}$ blood flow rate crossing Medium I, $\mathrm{m}^{3} / \mathrm{s}$

$\dot{q}_{I I}$ blood flow rate crossing Medium II, $\mathrm{m}^{3} / \mathrm{s}$

$\dot{q}$ total blood flow rate crossing the articulation zone considered, $\mathrm{m}^{3} / \mathrm{s}$

$a_{I}$ blood flow rate fraction in Medium I

$a_{I I}$ blood flow rate fraction in Medium II

$V_{I}$ Medium I volume, $\mathrm{m}^{3}$

$V_{I I}$ Medium II volume, $\mathrm{m}^{3}$

$v_{I_{-} b}$ total volume of separated gas per $\mathrm{m}^{3}$ of target tissue

$v_{I_{-} b_{-} \text {max }}$ maximum volume of separated gas per $\mathrm{m}^{3}$ of target tissue, during and after decompression

$N_{I}(r)$ number of micronuclei per $\mathrm{m}^{3}$ of target tissue with an initial radius greater than $\mathrm{r}, \mathrm{m}^{-3}$

$N_{I_{-} \max }$ total number of micronuclei per $\mathrm{m}^{3}$ of target tissue initially present in Medium $\mathrm{I}, \mathrm{m}^{-3}$

$N_{I_{-} \text {tot }}$ total number of micronuclei per $\mathrm{m}^{3}$ of target tissue recruited during decompression, $\mathrm{m}^{-3}$ 


\section{ACCEPTED MANUSCRIPT}

$A_{I}$ coefficient of the exponential law describing the initial distribution of nuclei dimensions in Medium I, $\mathrm{m}^{-1}$

$R_{r}$ radius of a microbubble generated from a nucleus with an initial radius $\mathrm{r}, \mathrm{Pa}$

$r_{0}$ minimal initial radius for the nuclei recruited at a given time, $\mathrm{m}$

$r_{0 \_ \text {min }}$ minimal initial radius for the nuclei recruited during decompression, $\mathrm{m}$

$t_{r_{0_{-} \min }}$ time corresponding to the recruitment of nuclei with an initial radius greater than $r_{0_{-} \min }, \mathrm{s}$

$\alpha_{I}$ parameter of the nuclei recruitment law, $\mathrm{N} / \mathrm{m}$

$\phi_{I}$ parameter of the nuclei recruitment law

$\beta_{I}$ sum of the metabolic gases and the water vapour tension in Medium I

$\gamma_{I}$ surface tension of Medium I

$\mathfrak{R}$ perfect gas law coefficient, $\mathrm{Pa} \cdot \mathrm{m}^{3} / \mathrm{mol} / \mathrm{K}$

$T$ temperature in medium I, i.e. $310 \mathrm{~K}$

$K$ elastic bulk modulus in the target tissue, $\mathrm{Pa}$ 
Tables

\begin{tabular}{|c|c|c|}
\hline$A_{1}$ & $m^{-1}$ & \multirow{10}{*}{$\begin{array}{l}P \\
\mathrm{P} \\
\mathrm{y} \\
\mathrm{s} \\
\mathrm{i} \\
\mathrm{c} \\
\mathrm{a} \\
\mathrm{I}\end{array}$} \\
\hline $\mathrm{N}_{\mathrm{I} \text { max }}$ & $m^{-3}$ & \\
\hline$\gamma_{1}$ & $\mathrm{~N} / \mathrm{m}$ & \\
\hline $\mathrm{K}$ & $\mathrm{Pa}$ & \\
\hline$\alpha_{1}$ & $\mathrm{~N} / \mathrm{m}$ & \\
\hline$\Phi_{1}$ & - & \\
\hline$S_{I, j} \quad j \in[1 ; N]$ & $\mathrm{mol} / \mathrm{m}^{3} / \mathrm{Pa}$ & \\
\hline$D_{l j} j \in[1 ; N]$ & $\mathrm{m}^{2} / \mathrm{s}$ & \\
\hline $\mathrm{S}_{\mathrm{II}, \mathrm{j}} \quad \mathrm{j} \in[1 ; \mathrm{N}]$ & $\mathrm{mol} / \mathrm{m}^{3} / \mathrm{Pa}$ & \\
\hline$D_{I I\lrcorner j} j \in[1 ; N]$ & $\mathrm{m}^{2} / \mathrm{s}$ & \\
\hline
\end{tabular}

\begin{tabular}{|c|c|c|}
\hline $\mathrm{k}_{\mathrm{l}, \mathrm{j}} \quad \mathrm{j} \in[1 ; \mathrm{N}]$ & $\min ^{-1}$ & $P$ \\
\hline $\mathrm{k}_{\mathrm{II}, \mathrm{j}} \quad \mathrm{j} \in[1 ; \mathrm{N}]$ & $\min ^{-1}$ & ${ }^{h} p$ \\
\hline$a_{1}$ & - & $\mathrm{s}^{\mathrm{a}} \mathrm{r}$ \\
\hline$a_{\|}$ & - & $a_{0}^{a}$ \\
\hline $\mathrm{k}_{\mathrm{d} \_\mathrm{ll}, \mathrm{j}} \quad \mathrm{j} \in[1 ; \mathrm{N}]$ & $\min ^{-1}$ & \\
\hline$\omega_{\mathrm{j}} \quad \mathrm{j} \in[1 ; \mathrm{N}]$ & - & ${ }_{g}^{0} t$ \\
\hline$\beta_{\mathrm{I}}$ & $\mathrm{N} / \mathrm{m}$ & $\mathrm{i}_{\mathrm{r}}^{\mathrm{e}}$ \\
\hline$\Psi_{1}$ & $\mathrm{~m}^{2}$ & $\begin{array}{l}c \\
a\end{array}$ \\
\hline$\Psi_{\|}$ & $\mathrm{m}^{2}$ & 1 \\
\hline
\end{tabular}

Table 1 - Physical and physiological parameters of the model

\begin{tabular}{|c|c|c|c|c|}
\cline { 3 - 5 } \multicolumn{2}{c|}{} & $\begin{array}{c}\text { Solubility } \\
\left(\mathrm{mol} / \mathrm{m}^{3} / \mathrm{Pa} @ 37^{\circ} \mathrm{C}\right)\end{array}$ & $\begin{array}{c}\text { Diffusion coefficient } \\
\left(\mathrm{m}^{2} / \mathrm{s} @ 37^{\circ} \mathrm{C}\right)\end{array}$ & $\begin{array}{c}\text { Permeability } \\
\left(\mathrm{mol} / \mathrm{m} / \mathrm{Pa} / \mathrm{s} @ 37^{\circ} \mathrm{C}\right)\end{array}$ \\
\hline \multirow{2}{*}{ Water, plasma } & Nitrogen & $5.1 \mathrm{E}-06$ & $2.2 \mathrm{E}-09$ & $1.1 \mathrm{E}-14$ \\
\cline { 2 - 5 } & Helium & $3.3 \mathrm{E}-06$ & $6.3 \mathrm{E}-09$ & $2.1 \mathrm{E}-14$ \\
\hline \multirow{2}{*}{ Oil } & Nitrogen & $2.9 \mathrm{E}-05$ & $7.0 \mathrm{E}-10$ & $2.0 \mathrm{E}-14$ \\
\cline { 2 - 5 } & Helium & $6.4 \mathrm{E}-06$ & $1.9 \mathrm{E}-09$ & $1.2 \mathrm{E}-14$ \\
\hline \multirow{2}{*}{ Lipid } & Nitrogen & $2.4 \mathrm{E}-05$ & $1.0 \mathrm{E}-09$ & $2.4 \mathrm{E}-14$ \\
\cline { 2 - 5 } & Helium & $5.8 \mathrm{E}-06$ & $2.8 \mathrm{E}-09$ & $1.6 \mathrm{E}-14$ \\
\hline
\end{tabular}

Table 2 - Physical properties considered for nitrogen and helium : solubility coefficient, diffusion coefficient and permeability - extracted from (8) 


\begin{tabular}{|c|c|c|}
\hline$A_{I}$ & $\mathrm{~m}^{-1}$ & {$\left[5 \times 10^{5} ; 5 \times 10^{6}\right]$} \\
\hline$N_{I_{-} \max }$ & $\mathrm{m}^{-3}$ & {$\left[10^{6} ; 10^{12}\right]$} \\
\hline$K$ & $\mathrm{~Pa}$ & {$\left[10^{4} ; 10^{7}\right]$} \\
\hline$\alpha_{I}$ & $\mathrm{~N} / \mathrm{m}$ & {$[0.09 ; 0.1]$} \\
\hline$k_{I, j}, k_{I I, j}, \mathrm{j} €[1 ; \mathrm{N}]$ & $\min ^{-1}$ & {$[0.001 ; 0.01]$} \\
\hline$k_{d_{-} I I, j}, \mathrm{j} €[1 ; \mathrm{N}]$ & $\min ^{-1}$ & {$[0.001 ; 0.1]$} \\
\hline$\omega_{j}, \mathrm{j} €[1 ; \mathrm{N}]$ & - & {$[0.1 ; 10]$} \\
\hline$\psi_{I}, \psi_{I I}$ & $\mathrm{~m}^{2}$ & {$\left[10^{-6} ; 10^{-5}\right]$} \\
\hline Lipid percentage in Medium I and Medium II & - & {$[0 ; 1]$} \\
\hline
\end{tabular}

Table 3 - Range of values considered for model parameters during the correlation phase 


\begin{tabular}{|c|c|c|c|}
\hline Parameter & Unit & Set A & Set B \\
\hline$A_{I}$ & $\mathrm{~m}^{-1}$ & $10^{6}$ & $10^{6}$ \\
\hline$N_{I_{-} \max }$ & $\mathrm{m}^{-3}$ & $5 \times 10^{10}$ & $5 \times 10^{10}$ \\
\hline$K$ & $\mathrm{~Pa}$ & $3 \times 10^{6}$ & $3 \times 10^{6}$ \\
\hline$\alpha_{I}$ & $\mathrm{~N} / \mathrm{m}$ & 0.1 & 0.1 \\
\hline$k_{I, N_{2}}, k_{I I, N_{2}}$ & $\min ^{-1}$ & $0.0010,0.0020$ & $0.0010,0.0015$ \\
\hline$k_{I, \mathrm{He}}, k_{I I, \mathrm{He}}$ & $\min ^{-1}$ & $0.0010,0.0025$ & $0.0010,0.0030$ \\
\hline$k_{d_{-} I I, N_{2}}, k_{d_{-} I I, H e}$ & $\min ^{-1}$ & $0.008,0.028$ & $0.007,0.029$ \\
\hline$\omega_{N_{2}}, \omega_{H_{e}}$ & - & $8,6.3$ & $8,4.0$ \\
\hline$\psi_{I}, \psi_{I I}$ & $\mathrm{~m}^{2}$ & $5 \times 10^{-6}, 7.5 \times 10^{-6}$ & $2.5 \times 10^{-6}, 6.5 \times 10^{-6}$ \\
\hline $\begin{array}{c}\text { Lipid percentage in } \\
\text { Medium I and Medium II }\end{array}$ & - & $0 \%, 10 \%$ & $0 \%, 45 \%$ \\
\hline
\end{tabular}

Table 4 - Set of parameters deduced from the correlation phase

\begin{tabular}{|c|c|c|c|c|}
\hline & \multicolumn{2}{|c|}{$T_{1 / 2}(\min )$ Set $A$} & \multicolumn{2}{|c|}{$T_{1 / 2}(\min )$ Set $B$} \\
\hline & $\mathrm{N}_{2}$ & $\mathrm{He}$ & $\mathrm{N}_{2}$ & $\mathrm{He}$ \\
\hline & \multicolumn{4}{|c|}{ Bottom } \\
\hline Medium I & 85 & 85 & 85 & 85 \\
\hline Medium II & 45 & 35 & 55 & 30 \\
\hline 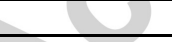 & \multicolumn{4}{|c|}{ Decompression } \\
\hline Medium I & 230 & 230 & 230 & 230 \\
\hline Medium II & 115 & 90 & 155 & 75 \\
\hline
\end{tabular}

Table 5 - Half-times chosen after correlation for nitrogen and helium, at bottom level and during the decompression 
A new biophysical decompression model for estimating the risk of articular bends during and after

\section{decompression}

\section{Highlights}

$>$ We present a biophysical decompression model for describing bends occurrence

$>$ A joint is broken into two parts exchanging inert gases by perfusion and diffusion

$>$ The diffusion pathway allows the slow amplification of bubbles during decompression

$>$ A risk database extracted from COMEX experience is used for the model correlation

$>$ The risk of bends occurrence is found to be linked to the bubbles volume formed 


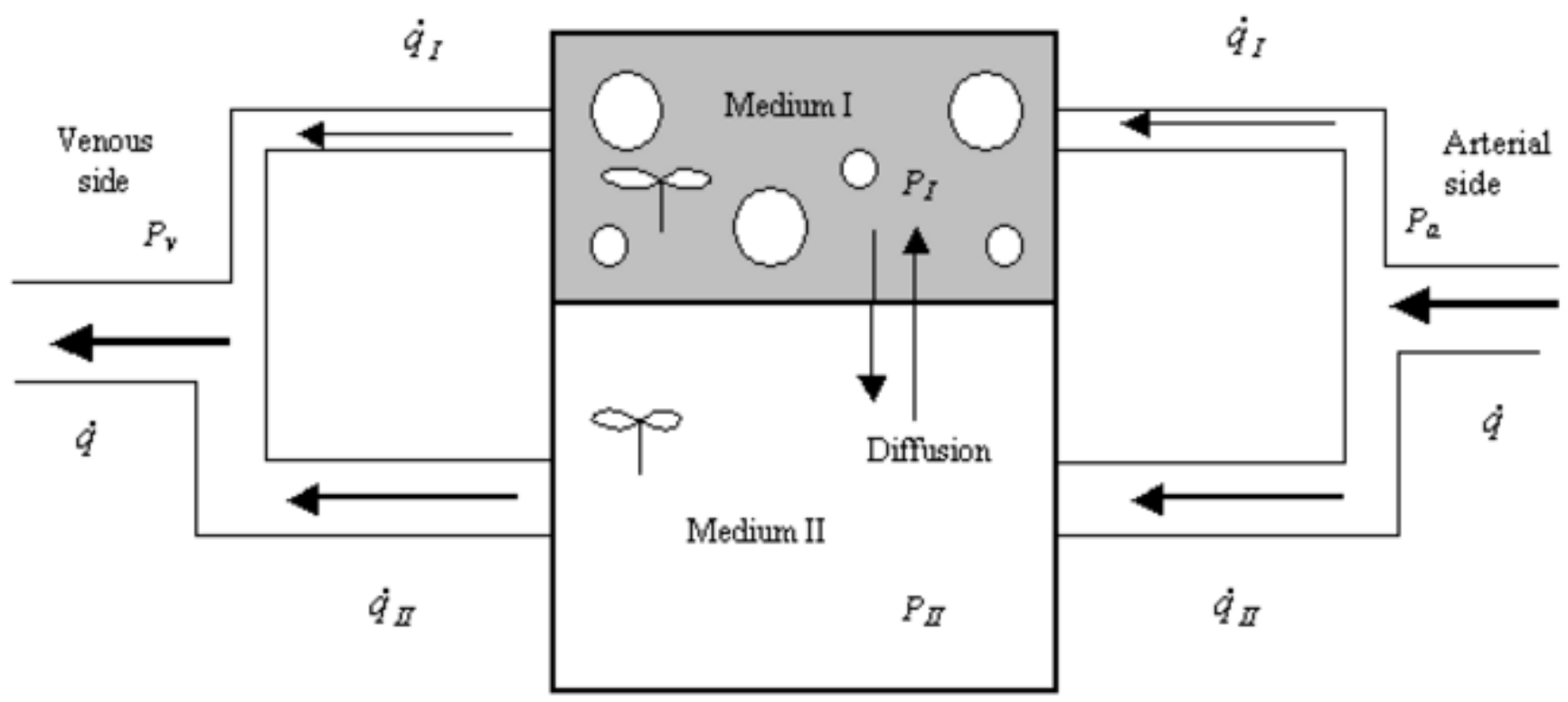




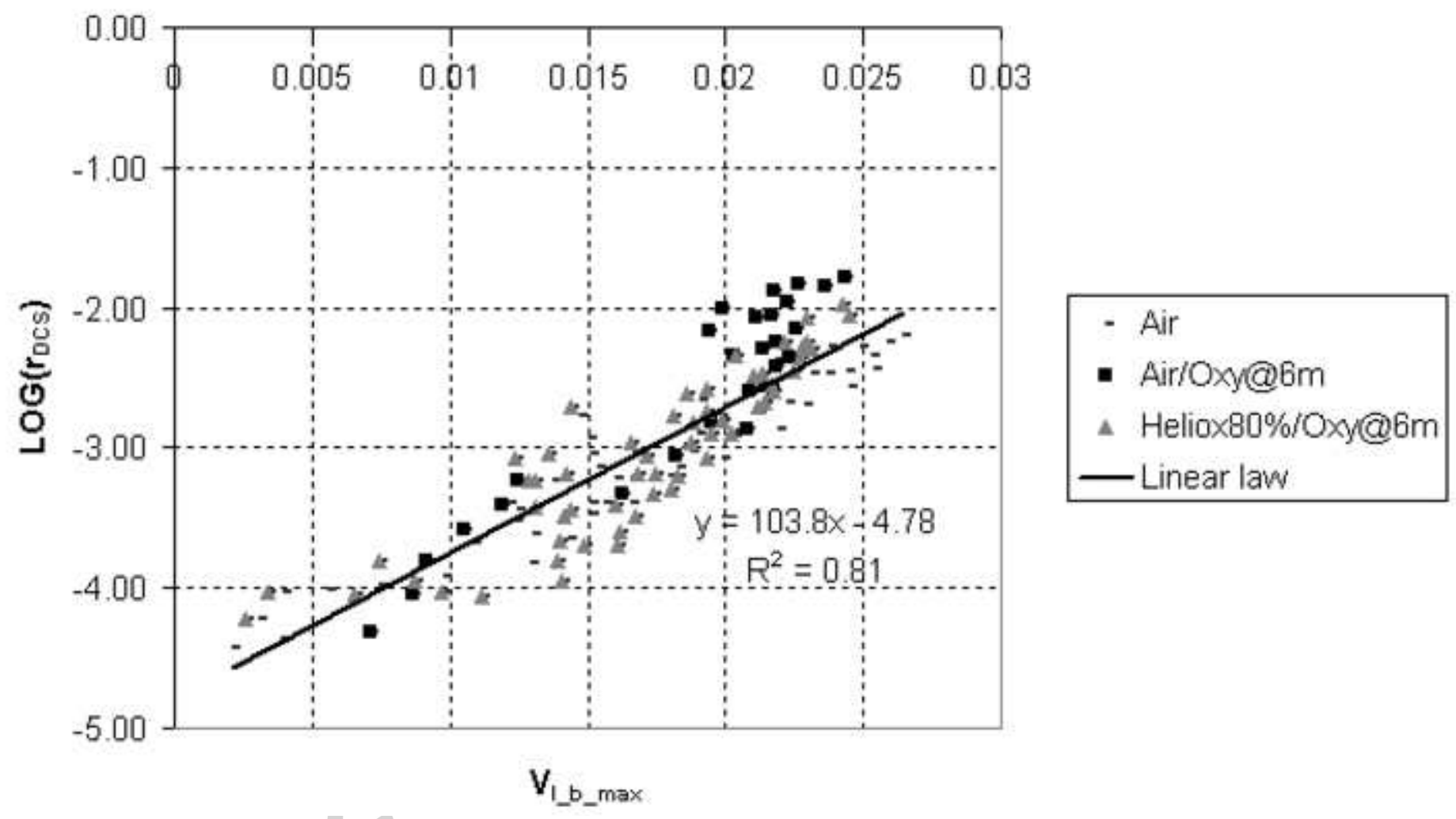




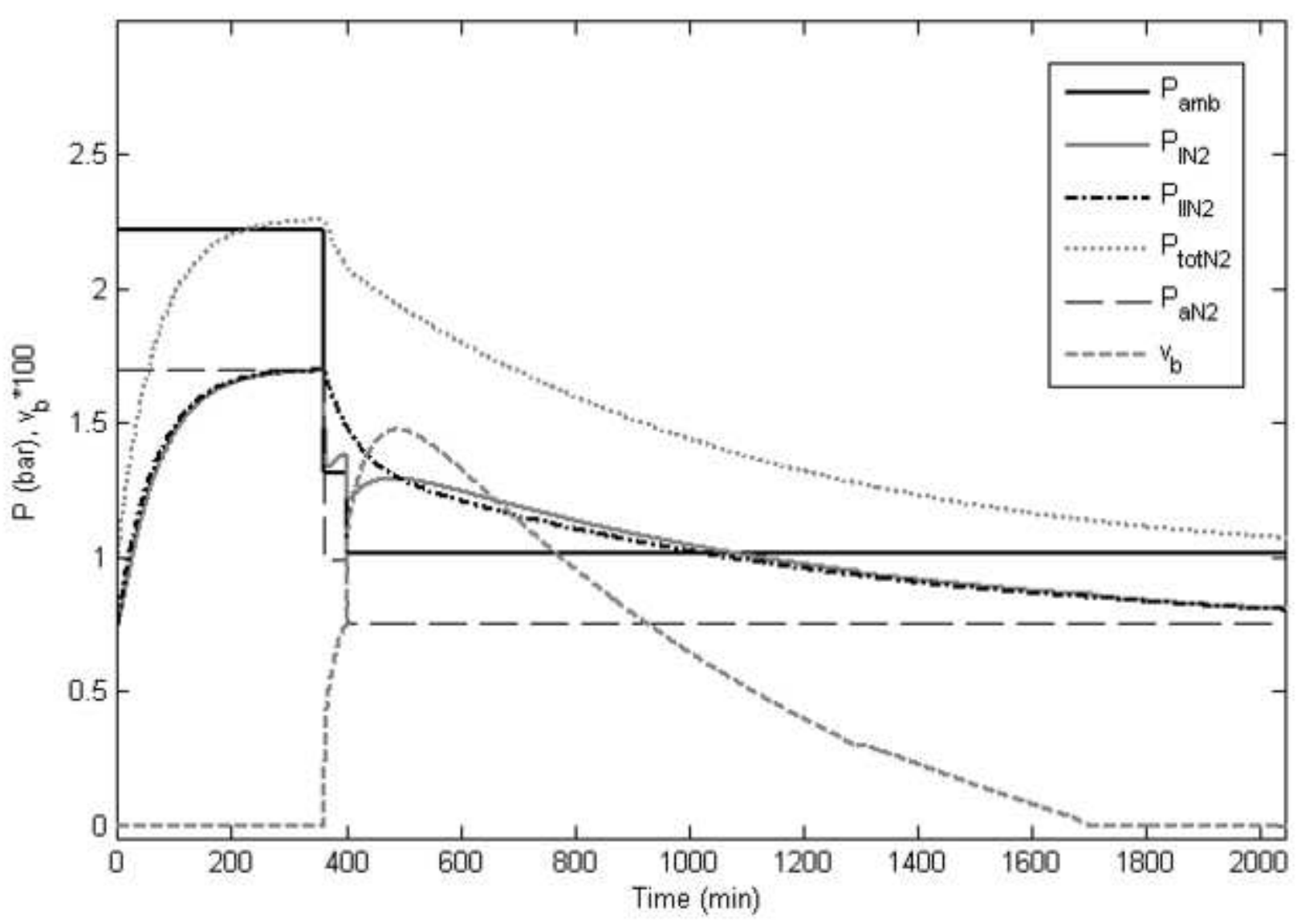




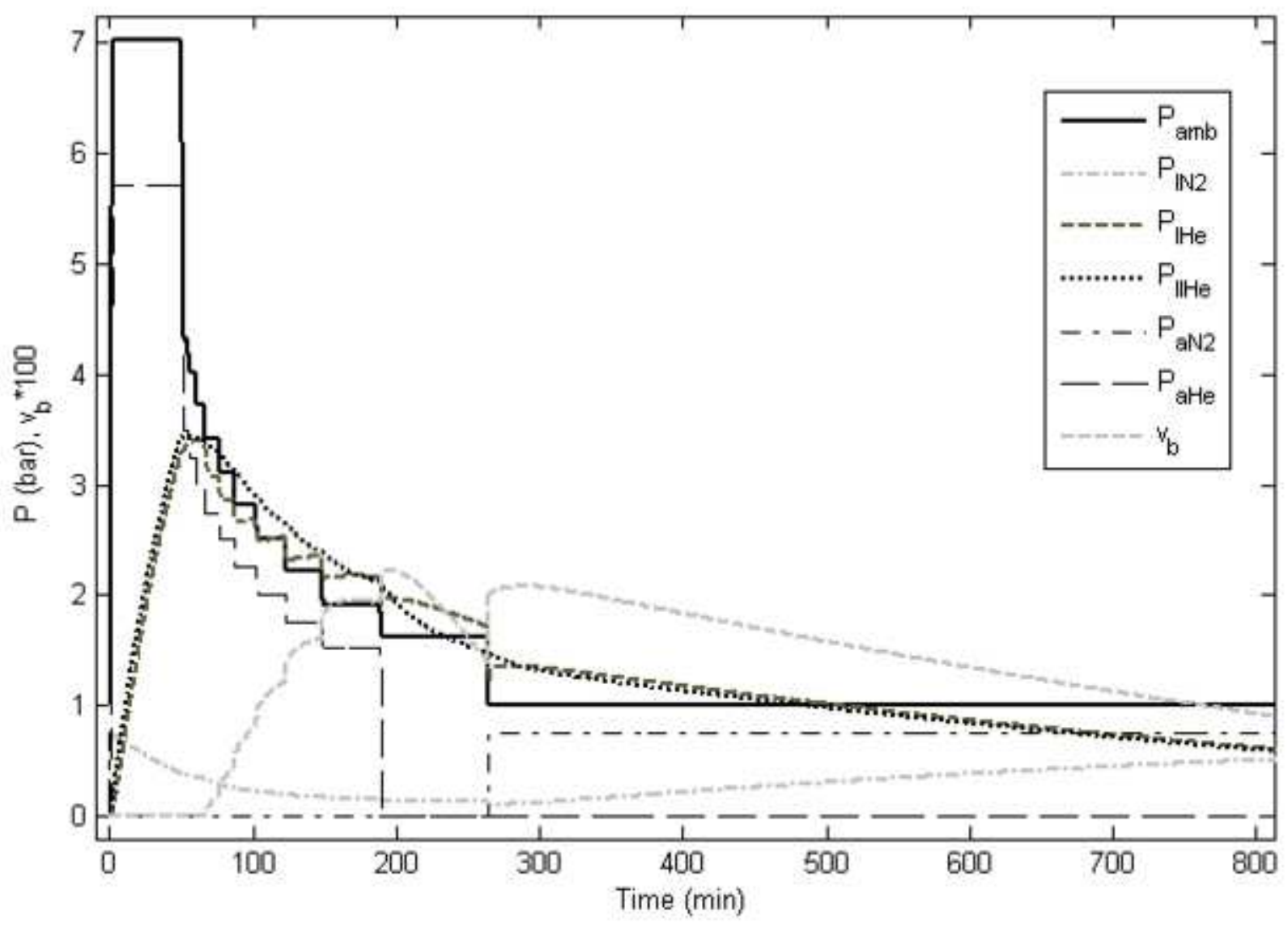




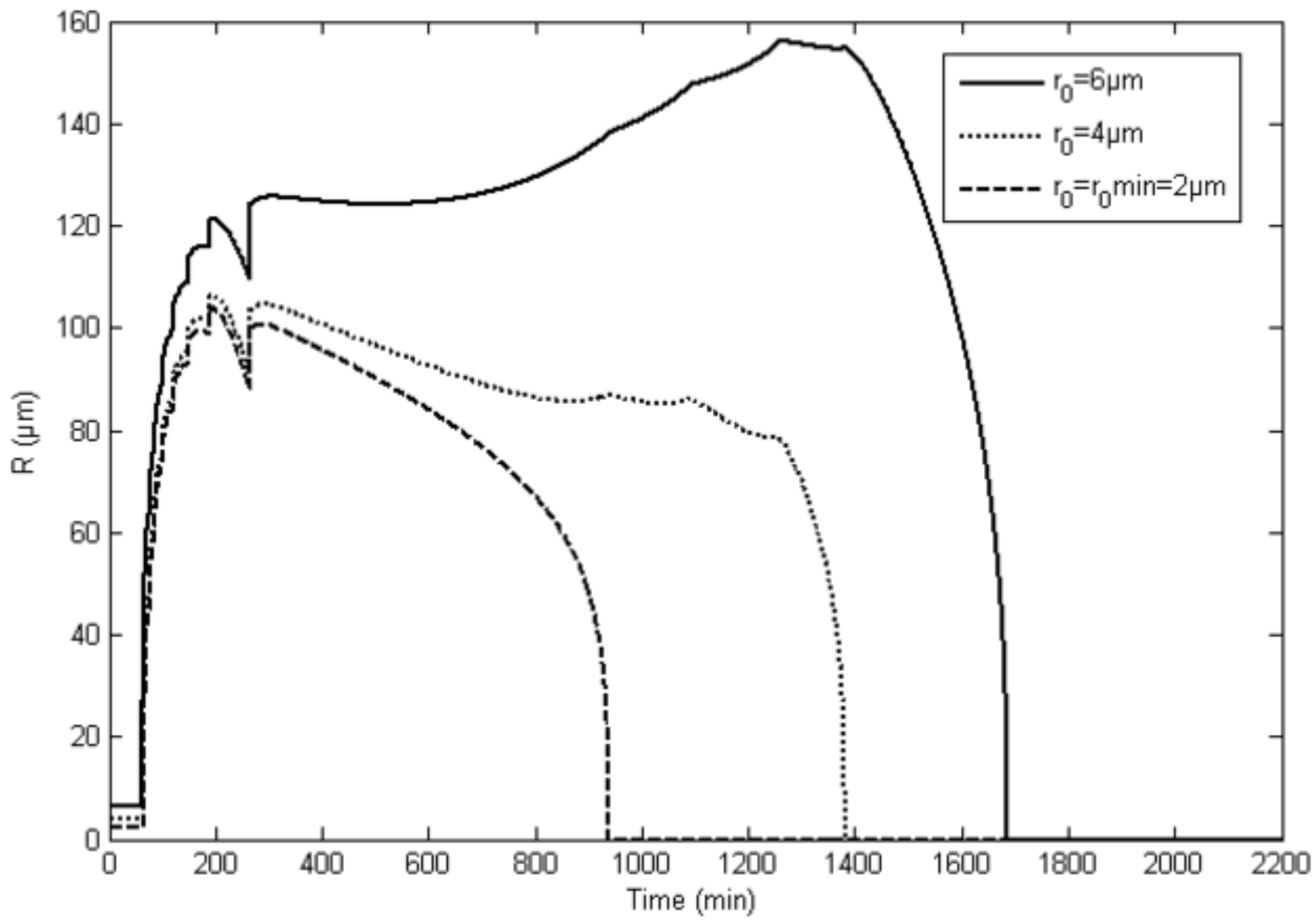




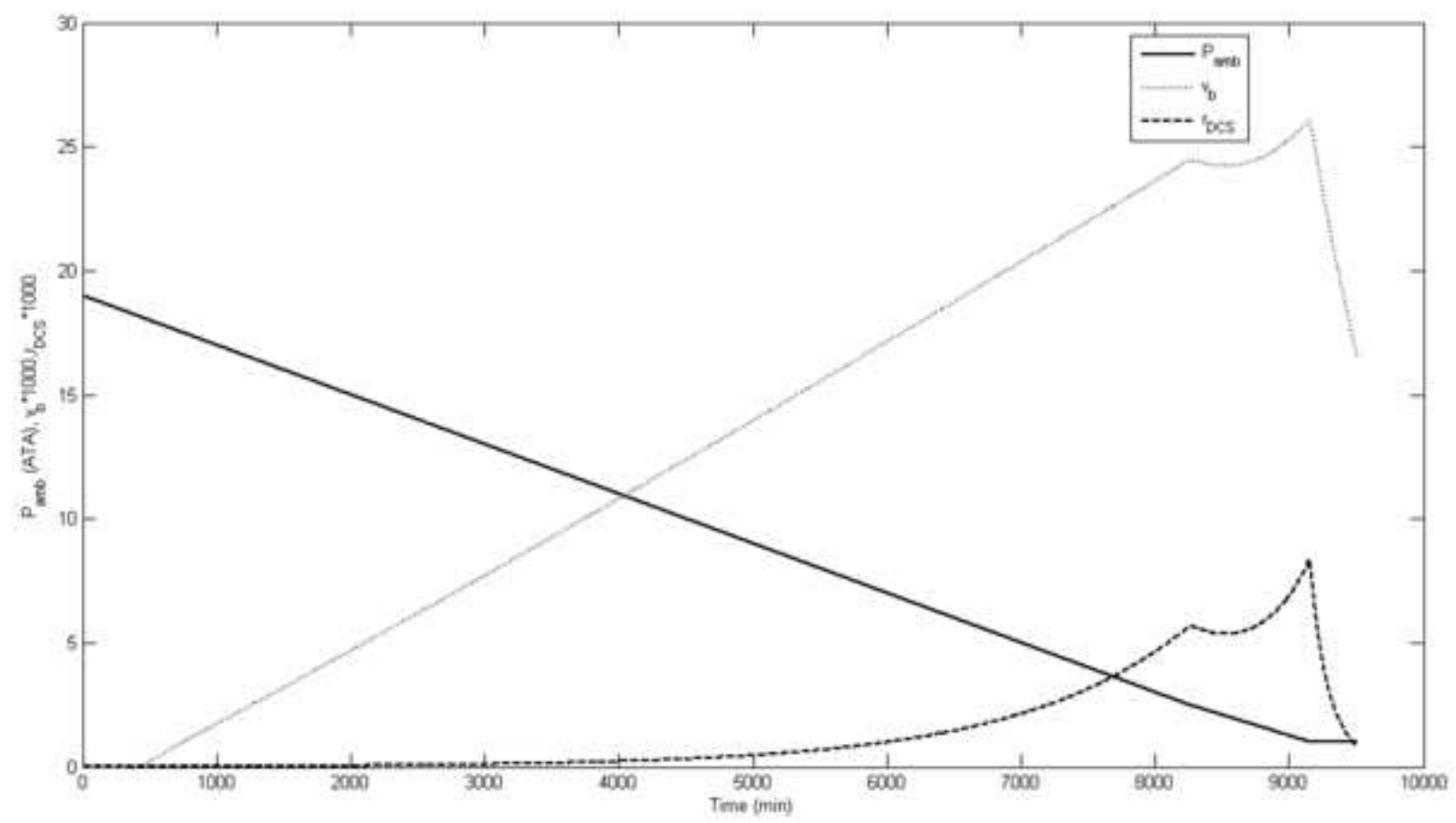




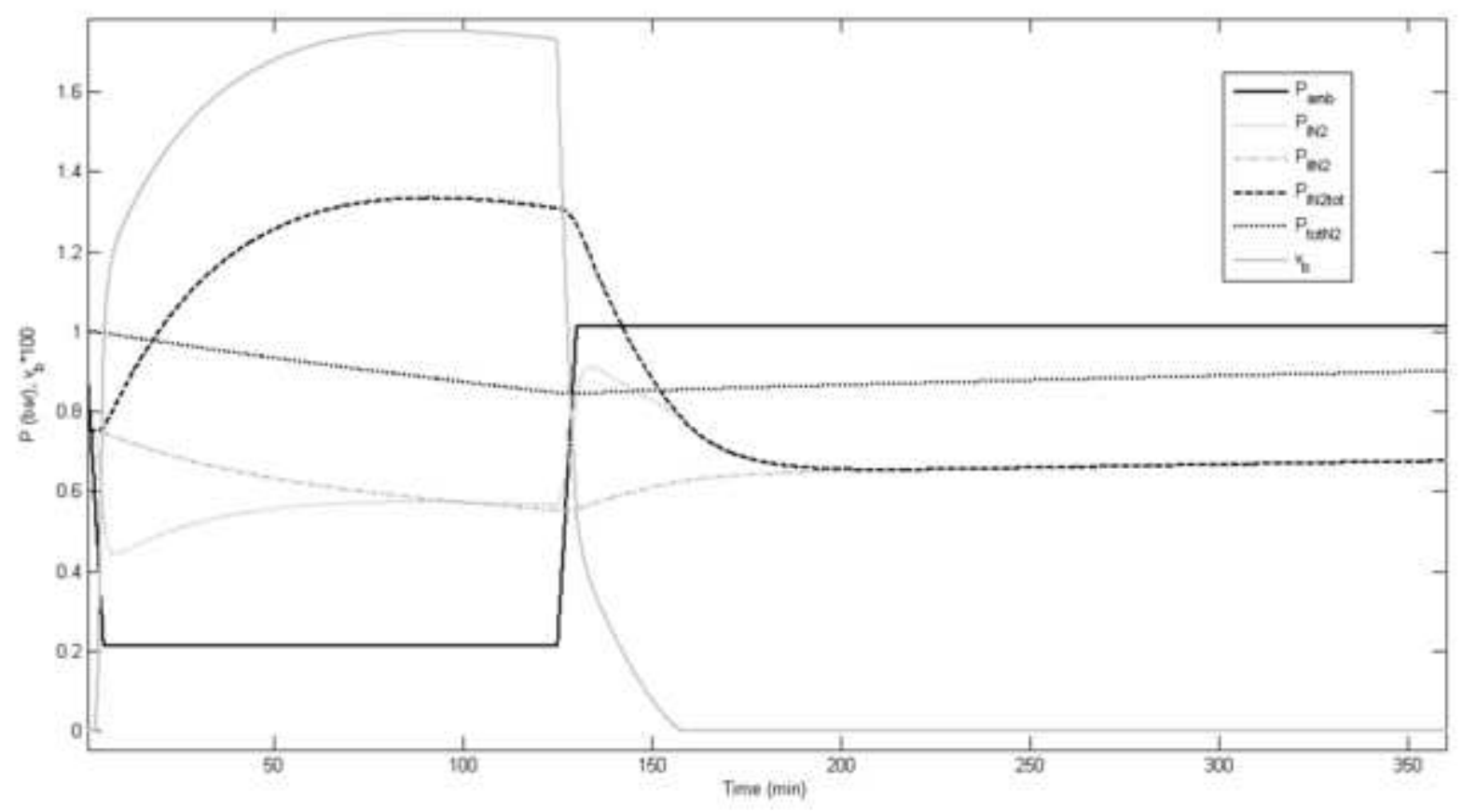

